\title{
Pore-scale study of thermal fields during evaporation from drying porous surfaces
}

\author{
Milad Aminzadeh ${ }^{1, *}$ and Dani Or ${ }^{1}$ \\ ${ }^{1}$ Department of Environmental Systems Science, ETH Zürich, Zürich, Switzerland \\ *Corresponding Author: milad.aminzadeh@usys.ethz.ch
}

\begin{abstract}
The drying of a porous surface and subsequent pore emptying resemble a sequence of drainage whereby pores are invaded according to their respective capillary size (large pores are invaded first). The emptying of pores modifies both the local evaporative fluxes and surface thermal fields adjacent to invaded pores. These local adjustments could result in a gradual decrease in evaporation rate and a potential increase in surface temperature thereby altering energy partitioning over the drying surface as described by the Pore-scale Coupled Energy Balance (PCEB) model of Aminzadeh and Or (2014). This study aims to observe and quantify these dynamic pore-scale mass and energy interactions to test key assumptions in the basis of the PCEB model and gain new insights into these important processes. We used a novel experimental system consisting of individual and clustered evaporating pores drilled into rough glass surfaces. Thermal fields around individual evaporating pores and their interactions were observed using highly resolved infrared imager under different radiative fluxes. Thermal observations and direct measurements of evaporative fluxes were in good agreement with predictions by the PCEB model. Drying of a glass beads surface was captured by optical and thermal imaging to provide links between pore emptying sequence and surface thermal adjustments. The results highlight the upscalability of pore-based representation of surface drying and the predictability of energy partitioning over drying porous surfaces.
\end{abstract}

Keywords: Porous surface; Evaporative drying; Infrared thermography; Surface temperature; Energy balance 


\section{Introduction}

During gradual drying of initially saturated porous media surface pores are sequentially invaded by the air phase from largest to smallest [1,2] in a process resembling drainage [3]. The resulting local capillary pressure gradients induce liquid flow from the invaded larger pores to liquid filled smaller pores with menisci anchored at the surface [1,4] until smallest pores at the surface are ultimately invaded marking the end of stage 1 evaporation. The socalled stage 1 evaporation is characterized by a relatively constant evaporative flux where capillary transport sustains continuous liquid pathways from the receding drying front within the porous medium to the vaporization plane at the surface [5]. At a certain drying front depth termed the evaporative characteristic length by Lehmann et al. [6], hydraulic continuity with the surface is interrupted and subsequently the primary vaporization plane migrates below the surface. The migration of vaporization plane below the surface marks the onset of diffusion controlled stage 2 evaporation that is often accompanied by a significant reduction in evaporative flux $[2,7-10]$.

For a uniform spatial distribution of pore sizes on the surface, the gradual drying of surface results in an increase in the average spacing between remaining actively evaporating pores. This increased spacing affects the evaporative flux from remaining evaporating pores through a nonlinear adjustment of diffusive resistance to vapor transport across the air boundary layer that maintains a nearly constant evaporative flux during stage 1 evaporation [10-13]. The capacity of a surface to maintain a nearly constant evaporation rate during stage 1 results in a nearly constant mean surface temperature reflecting the coupling between heat and mass transfer processes during surface drying [14,15]. Temperature dynamics of individual evaporating capillaries including variation of temperature gradients beneath the meniscus have been widely studied [16-20]. Sefiane and Ward [21] have used infrared thermography to show a significant increase of meniscus temperature due to the reduction of evaporative flux 
as it recedes into a capillary. Such local changes in surface temperature during pore emptying are in the core of the Pore-scale Coupled Energy Balance (PCEB) model of Aminzadeh and Or [22]. The pore-based representation of vapor and heat fluxes from drying surfaces seeks to quantify thermal fields around invaded pores that modify lateral conductive heat fluxes and thus shape mean surface temperature.

The PCEB model links variations of evaporative flux as surface pores gradually empty with adjustments of surface temperature hence providing a mechanistic (and analytical) framework for prediction of energy partitioning over drying porous surfaces. The complex adjustments of evaporative and thermal fields around individual surface pores are represented using a porecentered unit cell in which surface gradual drying is translated to increased spacing (dry area) around the representative pore. The PCEB solves the coupled steady state heat and mass transfer equations to quantify evaporative flux and corresponding temperature distribution on the surface of unit cell around the representative pore and then average it to represent mean temperature of the drying surface. Experimental evaluations of the PCEB model for prediction of macroscopic surface energy balance components $[15,22]$ have demonstrated the great potential of such pore-centered modeling approach. Nevertheless, key ingredients and assumptions of the PCEB model have not been systematically tested such as the thermal fields forming around individual and clusters of pores, the upscalability of pore-based representation of energy balance, the sensitivity of surface temperature to pore emptying sequences and more. We thus designed evaporation experiments using high resolution infrared imaging to assess the formation of lateral thermal fields around individual pores (and pore clusters) and the steady state representation of gradual surface drying in which the temporal evolution of microscopic thermal adjustments are considered as a sequence of stepwise variations of surface saturation. 
The specific objectives of this study are: (1) to experimentally test key elements and assumptions in the unit cell used in PCEB model by comparing observed temperature distributions around individual and clusters of pores and associated evaporative flux with model predictions, (2) to investigate the effect of pore spacing and radiative flux on the perpore vapor diffusion and formation of interacting surface thermal fields using microscopic infrared thermography, (3) to systematically observe dynamics of microscopic thermal adjustments and evaluate the assumption of steady state representation of surface drying resolved by variation of surface liquid content, and (4) to link the pore-scale thermal adjustments around individual pores with evaporative pore emptying process during sequential invasion of surface pores.

Following a brief introduction of the PCEB model, details of the experimental system used to study thermal fields around individual pores and porous glass beads surfaces are presented. The experimental results for a range of external conditions are compared with PCEB model predictions of temperature and evaporative fluxes. Finally, the pore emptying sequence of glass beads sample and evolution of thermal fields are observed by optical and thermal imaging and the upscalability of the pore-centered approach for surface energy partitioning is assessed.

\section{The Pore-scale Coupled Energy Balance (PCEB) model}

The coupling between surface temperature and evaporative flux during gradual drying of a porous medium has been analytically studied by the Pore-scale Coupled Energy Balance (PCEB) model [22]. The physically based modeling framework offers unique capabilities for prediction of surface energy balance components in hydrological applications. It obviates the need for empirical resistances to heat and mass exchanges between evaporating surfaces and air by linking intrinsic resistance to vapor diffusion from discrete pores (whose spacing 
increases with surface drying) with microscopic thermal changes around individual surface pores.

To simplify the complex dynamics of sequential invasion of surface pores during evaporative drying, the PCEB considers a unit cell comprised of a characteristic evaporating pore surrounded by non-evaporating surface around it. The ratio of pore area to the unit cell area defines the surface liquid content under consideration. Accordingly, the gradual surface drying is translated to an increase in the dry region around representative pore in the unit cell (reduction of surface liquid content). This increase affects both diffusive vapor flux [12] and mean surface temperature of the unit cell that are analytically determined assuming steady state conditions for each surface liquid content. This pore-scale approach allows us to systematically investigate the coupling between diffusive vapor transfer across the air boundary layer and evolution of thermal fields around surface pores by variation of dry area around the unit cell's pore representing the effect of surface drying on the spacing around remaining wet pore on the surface.

The PCEB considers components of the surface energy balance for an infinitesimal element of solid region around the representative pore including lateral conductive heat flux, long wave and shortwave radiation, convective heat flux between surface and air flow (sensible heat flux), and vertical conductive flux towards (or from) the surface which lead to the following differential equation for the surface temperature distribution around the pore:

$$
\frac{k}{r} \frac{\partial}{\partial r}\left(r \frac{\partial T}{\partial r}\right)+\frac{1}{\Delta h}\left(\sigma \varepsilon_{a} T_{a}^{4}-\sigma \varepsilon_{s} T^{4}+R_{S}+h_{a}\left[T_{a}-T\right]+\frac{k}{\Delta H}\left[T_{s}-T\right]\right)=0 \quad a \leq r \leq b
$$

where $T$ is the unit cell temperature $[\mathrm{K}]$ at radial coordinates $r[\mathrm{~m}], k$ is the effective thermal conductivity of the medium $[\mathrm{W} / \mathrm{mK}], R_{S}$ is the net incoming shortwave radiation flux $\left[\mathrm{W} / \mathrm{m}^{2}\right], T_{a}$ is the ambient air temperature $[\mathrm{K}], \sigma$ is the Stefan-Boltzmann constant [ 
$\left.5.67 \times 10^{-8} \mathrm{~W} / \mathrm{m}^{2} \mathrm{~K}^{4}\right], \varepsilon_{s}$ is the surface emissivity, $\varepsilon_{a}$ is the atmospheric emissivity, $\Delta h$ is the thickness of surface evaporating pore [m] (unit cell) that is assumed as a single grain size, $\Delta H$ is the thermal decay depth below the surface [m] [23] and $T_{s}$ is the linearized vertical temperature [K] at $\Delta H[22,23]$. In Eq. (1), $h_{a}$ is the mean convective heat transfer coefficient between the surface and air flow $\left[\mathrm{W} / \mathrm{m}^{2} \mathrm{~K}\right]$ that is quantified based on the air flow regime (laminar or turbulent) over a flat surface with finite characteristic length of $l$ using average Nusselt number as [24]:

$$
\begin{aligned}
& \overline{N u}=\frac{h_{a} l}{k_{a}}=0.664 \operatorname{Re}_{l}^{1 / 2} \operatorname{Pr}^{1 / 3} \quad \text { (laminar air flow) } \\
& \overline{N u}=\frac{h_{a} l}{k_{a}}=0.037 \operatorname{Re}_{l}^{4 / 5} \operatorname{Pr}^{1 / 3} \quad \text { (turbulent air flow) }
\end{aligned}
$$

where $l$ is the characteristic length of the surface in principle direction of air flow, $k_{a}$ is the heat conduction coefficient of air, and $R e$ and $\operatorname{Pr}$ are the Reynolds and Prandtl numbers, respectively. Considering the small size of unit cell, air temperature and convective heat transfer coefficient are assumed constant over the surface.

A key aspect of the PCEB model is the definition of boundary conditions for the energy balance equation (Eq. 1). Considering the interaction between thermal fields around adjacent pores we define a zero heat flux at the border of unit cell $\left(\left.\frac{\partial T}{\partial r}\right|_{r=b}=0\right.$, with $b$ as the radius of dry region). In addition, evaporation cooling within the pore results in a lateral conductive heat flux across the pore wall which defines the boundary condition between liquid and solid phases $\left(\left.\frac{\partial T}{\partial r}\right|_{r=a}=\frac{q_{c o n d}}{k}\right.$, with $a$ as the pore radius). Solution of energy balance equation with the described boundary conditions yields the following equation for temperature distribution around the evaporating pore at the surface: 


$$
T(r)=\frac{q_{\text {cond }}}{k \sqrt{\psi}} \times \frac{\left[\frac{K_{1}(\sqrt{\psi} b)}{I_{1}(\sqrt{\psi} b)} I_{0}(\sqrt{\psi} r)+K_{0}(\sqrt{\psi} r)\right]}{\left[\frac{K_{1}(\sqrt{\psi} b)}{I_{1}(\sqrt{\psi} b)} I_{1}(\sqrt{\psi} a)-K_{1}(\sqrt{\psi} a)\right]}+\varphi \quad a \leq r \leq b
$$

where $I_{0}$ and $I_{1}$, and $K_{0}$ and $K_{1}$ are the modified Bessel functions of the first and second kind, respectively, $q_{\text {cond }}$ is the conductive heat flux across the pore wall which is a function of temperature, and the argument $\psi$ and parameter $\varphi$ are defined as:

$$
\begin{gathered}
\psi=\frac{4 \sigma \varepsilon_{s} T_{a}^{3}+h_{a}+\frac{k}{\Delta H}}{k \Delta h} \\
\varphi=\frac{\sigma\left(\varepsilon_{a}+3 \varepsilon_{s}\right) T_{a}^{4}+h_{a} T_{a}+R_{S}+\frac{k}{\Delta H} T_{s}}{4 \sigma \varepsilon_{s} T_{a}^{3}+h_{a}+\frac{k}{\Delta H}}
\end{gathered}
$$

The conductive heat flux across the pore wall is quantified based on the energy balance for pore liquid near the surface as:

$$
q_{\text {cond }} \times 2 \pi a \Delta h=\left(q_{\text {evap }}-\left[q_{\text {rad }}+q_{\text {conv }}+G\right]\right) \times \pi a^{2}
$$

where $q_{\text {rad }}$, and $q_{\text {conv }}$ are radiative and convective flux on the surface of the pore, $G$ is the vertical conductive flux (e.g., soil heat flux) and $q_{\text {evap }}$ represents evaporative flux from pore surface that is characterized based on the vapor concentration difference:

$$
q_{\text {evap }}=C_{1}\left(C_{s}\left(\left.T\right|_{r=a}\right)-C_{a}\right)
$$

in which $C_{s}$ is vapor concentration at the surface of pore $\left[\mathrm{kg} / \mathrm{m}^{3}\right]$ that is quantified at a mean temperature representing the temperature distribution on the surface of pore liquid estimated as unit cell temperature at $r=a, C_{a}$ is vapor concentration in air mass $\left[\mathrm{kg} / \mathrm{m}^{3}\right]$, and parameter $C_{1}$ represents the mass transfer coefficient quantified based on the Schlünder [11] vapor diffusion model as: 


$$
C_{1}=\frac{D_{a} L / \theta}{\delta_{m}+\frac{2 a}{\pi} \sqrt{\frac{\pi}{4 \theta}}\left[\sqrt{\frac{\pi}{4 \theta}}-1\right]}
$$

where $\theta$ is the surface liquid content $\left[\mathrm{m}^{3} / \mathrm{m}^{3}\right], D_{a}$ is the vapor diffusion coefficient in air $\left[\mathrm{m}^{2} / \mathrm{s}\right], L$ is the latent heat of vaporization $[\mathrm{J} / \mathrm{kg}]$, and $\delta_{m}$ is the mass boundary layer thickness that is linked to the aerodynamic boundary layer thickness $\left(\delta_{v}\right)$ through the Schmidt number ( $S c$ ) as [25]:

$$
\delta_{m}=S c^{-1 / 3} \delta_{v}
$$

in which thickness of the aerodynamic layer for laminar flow over a flat surface is given based on the Blasius solution as [26]:

$$
\delta_{v}=\frac{5 x}{\sqrt{\operatorname{Re}_{x}}}
$$

where $x$ is the distance from the leading edge of the surface. Alternatively, for turbulent flow over an evaporating (flat) surface the value of $\delta_{m}$ in Eq. (6) can be quantified based on the analysis of Haghighi and Or [27]:

$$
\delta_{m}=\frac{C v}{0.1 u}
$$

in which $v$ is the kinematic viscosity of air $\left[\mathrm{m}^{2} / \mathrm{s}\right], u$ is mean air velocity $[\mathrm{m} / \mathrm{s}]$, and $C=22$ is a dimensionless constant.

Originally, an iterative solution of energy and vapor diffusion equations (Eq. 3 and 5) was proposed to obtain the evaporative flux and temperature distribution on the surface of representative unit cell [15]. More recently, however, it was shown that linearization of saturated vapor concentration $\left(C_{s}\right)$ enables a closed-form solution for the temperature of an 
evaporating surface under prescribed atmospheric conditions [22] (see Appendix A for details).

Equations (3) and (5) will be employed to present temperature distribution on the surface of unit cell and diffusive vapor fluxes in PCEB model and systematically investigate thermal interactions between adjacent pores at the surface of drying porous media. In the following section, systematic experiments were designed to evaluate model performance at pore-scale for representation of evaporating surfaces with sparse and dense pore spacing under different boundary conditions and examine its ability to capture dynamics of microscopic thermal adjustments that shape mean surface temperature over drying porous media.

\section{Experimental considerations}

Single pores and clusters of pores were drilled into rough glass plates to investigate dynamics of diffusive vapor flux and interacting thermal fields around evaporating pores during evaporation process (Figure 1). The diameter of pores was $550 \pm 10 \mu \mathrm{m}$ and size of glass plates was $60 \times 40 \mathrm{~mm}^{2}$ with thickness of $1.8 \mathrm{~mm}$. A small liquid reservoir was connected to a cylindrical tank to supply evaporating pores at constant head during experiments (Figure 2). The cylindrical tank with inner diameter of $20 \mathrm{~mm}$ and height of $40 \mathrm{~mm}$ was filled with evaporating liquid (ethanol or water) and capped with a rubber stopper to ensure evaporation takes place only at the surface of drilled pores into the glass plate located on the reservoir. A thin needle was inserted into the rubber to resemble the Mariotte bottle principle and sustain a constant flow rate (considering evaporation rate from pores) from cylindrical tank to the liquid reservoir with depth of $7 \mathrm{~mm}$ below the glass plate. 

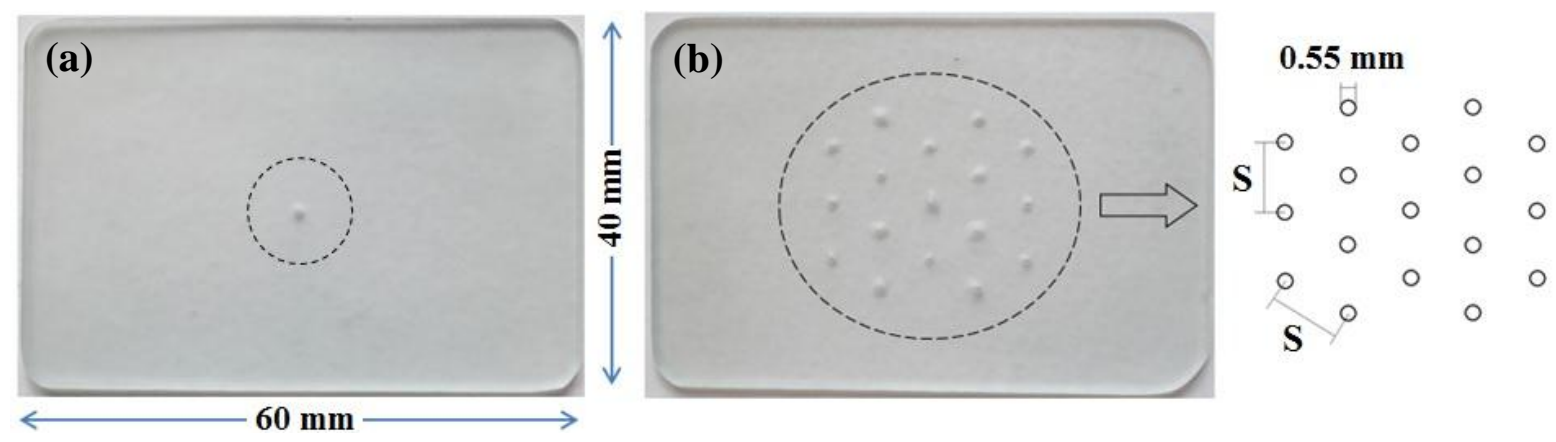

Figure 1. (a) A single pore (diameter $=0.55 \mathrm{~mm}$ ), and (b) a cluster of 17 pores (with diameter of $0.55 \mathrm{~mm}$ ) drilled into the roughened glass plates with surface area of $60 \times 40 \mathrm{~mm}^{2}$ and thickness of $1.8 \mathrm{~mm}$. Surfaces with different values of pore spacing (denoted by S) were considered in the study.

The resulting surface temperature during evaporation and interactions of thermal fields around individual surface pores were recorded using a sensitive thermal imager (FLIR SC6000, USA) with a modified and calibrated lens that enabled us to detect microscopic thermal signatures on evaporating surfaces at a spatial resolution of $640 \times 512$ pixels (see Shahraeeni and Or [28] for more information on IR thermography). The evaporative mass loss rate from surface pores was measured using a digital balance (KERN, ABT 220-5DM, Germany) with readability of $0.1 \mathrm{mg}$ and repeatability and linearity of 0.1 and $0.2 \mathrm{mg}$, respectively. Air temperature and relative humidity for water vapor $(R H)$ were monitored using an HMT337 sensor (Vaisala HUMICAP, HMT337, Finland) with stated accuracy of $0.1{ }^{\circ} \mathrm{C}$ and $1 \%$, respectively. We used a hotwire anemometer with $0.01 \mathrm{~m} / \mathrm{s}$ accuracy (Dostmann Electronic, P600, Germany) to measure mean air flow velocity over the sample generated by a small fan. A $150 \mathrm{~W}$ Tungsten lamp (Dedolight, Germany) was used to provide radiative flux over the sample (the spectrum similar to sunlight with a peak at $0.9 \mu \mathrm{m}$ due to the $3200 \mathrm{~K}$ temperature). The intensity of shortwave radiation over the surface was measured with a solar radiation power meter (Voltcraft, PL-110SM, Germany) with accuracy of $10 \mathrm{~W} / \mathrm{m}^{2}$. 


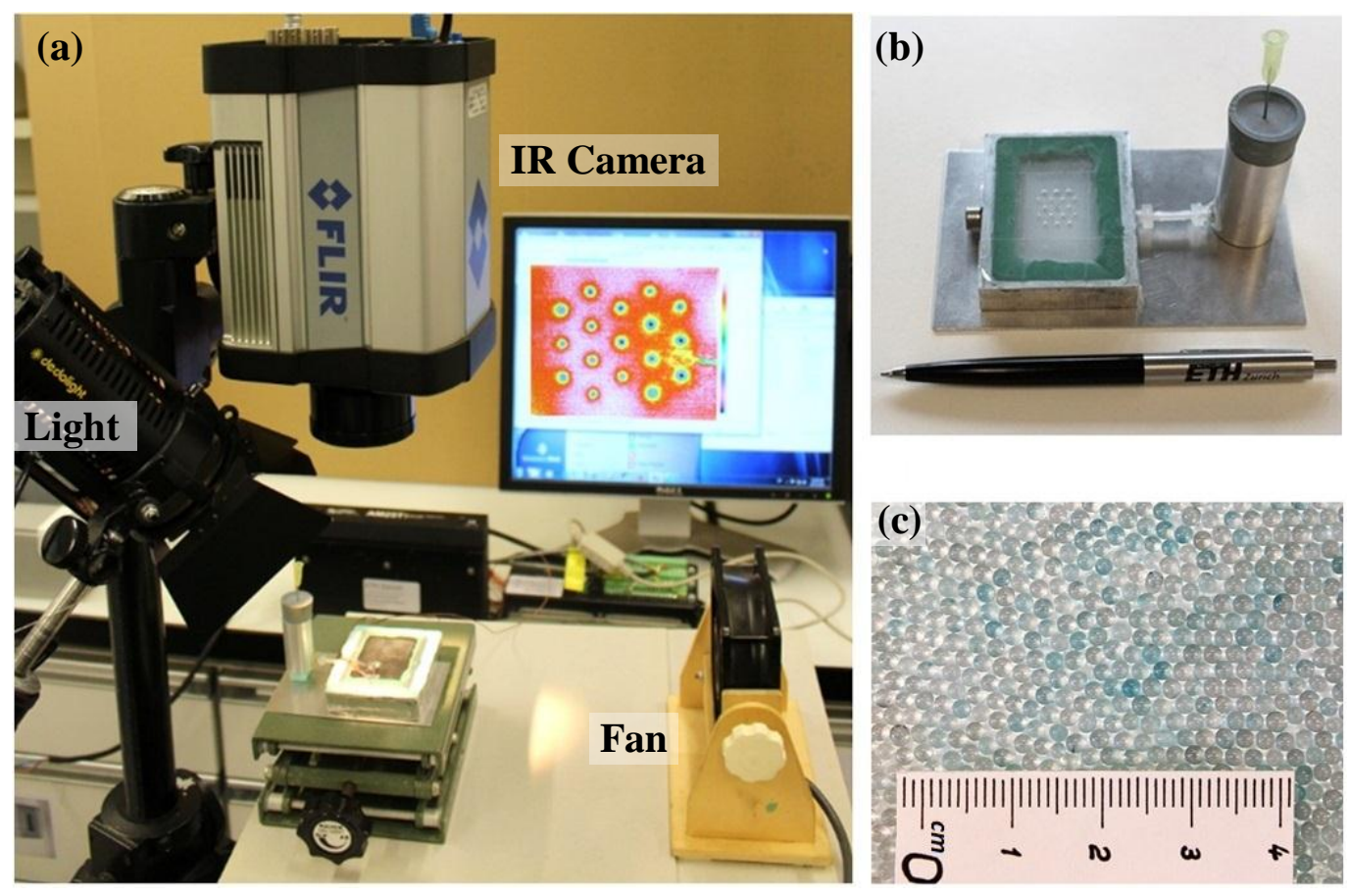

Figure 2. (a) Infrared thermography of evaporating surface using IR camera (FLIR SC6000), (b) drilled pores into the glass plate located on the reservoir; liquid is supplied to the reservoir from cylindrical tank considering the evaporative loss from surface pores, (c) monolayer glass beads surface $(1.6-2.4 \mathrm{~mm})$ on a glass plate.

A monolayer sample of glass beads on a thin glass plate with particle size ranging from 1.6 to $2.4 \mathrm{~mm}$ (Figure 2) was initially saturated to link evaporative pore emptying sequence at the surface of drying porous media with adjustment of thermal fields around individual surface pores. Pore-scale thermal adjustments on the surface of drying sample were recorded with infrared camera (at $10 \mathrm{~s}$ interval) while pore emptying sequence was monitored with a digital microscope (Dino-Lite, Taiwan). Degassed dye solution (brilliant blue) at a concentration of $0.04 \mathrm{~g} / \mathrm{l}$ was used to enhance visibility of the liquid phase.

In the following, results of microscopic thermography during evaporation experiments are presented and model predictions of pore-scale thermal interactions and evaporative flux are compared with measurements. 


\section{Results and discussion}

\subsection{Thermal field around an individual evaporating pore}

Figure 3 shows an example of thermal fields around individual evaporating pores that were observed by microscopic infrared thermography. The steady state IR images in Figure 3 were obtained during ethanol and water evaporation from individual pores (with diameter of 550 $\mu \mathrm{m})$ under different mean air velocity over the surface. Increasing air velocity decreases the thickness of air boundary layer and results in higher evaporative flux from the pore that is accompanied by lower surface temperature of the evaporating pore. For similar boundary conditions over the surface, the higher vapor pressure of ethanol relative to water resulted in higher evaporative fluxes and more pronounced surface temperature depression. Note that during evaporation experiments the pore was continually supplied to ensure it remains liquid filled with the meniscus anchored at the glass surface as assumed in the PCEB model. Evaporation cooling within the pore yields a conductive heat flux across the pore wall and shapes a sharp temperature gradient in solid region around the pore that is evident in IR images.

Comparison between measured radial temperature distribution around an evaporating pore from IR thermography and PCEB model predictions is depicted in Figure 4. The diffusive resistance in the pore-scale model expresses evaporating surface pore density in terms of surface liquid content (area of evaporating pores per unit surface area). For the special case of a single evaporating pore, the effective surface liquid content $\left(\theta_{e}\right)$ was determined for the radius at which the diffusive resistance reaches a constant value and thus per-pore evaporative flux remains constant with increasing dry region around the pore (decrease of $\theta$ ) (Figure 13b in Or et al. [10]). Hence, the effective liquid content representing the radius of unit cell in 
PCEB model (hypothetical pore spacing) is obtained from the derivative of pore evaporative flux $\left(q_{\text {evap }}\right.$ ) with respect to $\theta$ assuming constant boundary conditions over the surface as:

$$
\begin{gathered}
\frac{\partial q_{\text {evap }}}{\partial \theta}=0 \\
\Rightarrow \theta_{e}=\frac{a^{2}}{4 \pi \delta_{m}^{2}}=\frac{a^{2}}{r_{e}^{2}} \\
r_{e}=2 \sqrt{\pi} \delta_{m}
\end{gathered}
$$

where $a$ is the characteristic pore size (pore radius for circular pores), $r_{e}$ is the radius of unit cell corresponding to $\theta_{e}$, and $\delta_{m}$ is the thickness of mass boundary layer over the surface. Considering the range of air velocity in our experiments $(0.35$ to $4.5 \mathrm{~m} / \mathrm{s}), r_{e}$ varies from 15.2 to $4.25 \mathrm{~mm}$ for ethanol, and from 19 to $5.3 \mathrm{~mm}$ for water experiments.

Both PCEB model predictions and experimental results show relatively intense surface temperature gradients around individual evaporating pores that monotonically decrease with radial distance (Figure 4). In addition to thermal measurements, evaporation rates were also measured using a digital balance and results were in reasonable agreement with model predictions for different evaporating liquids (ethanol and water) and boundary conditions (Figure 5). 


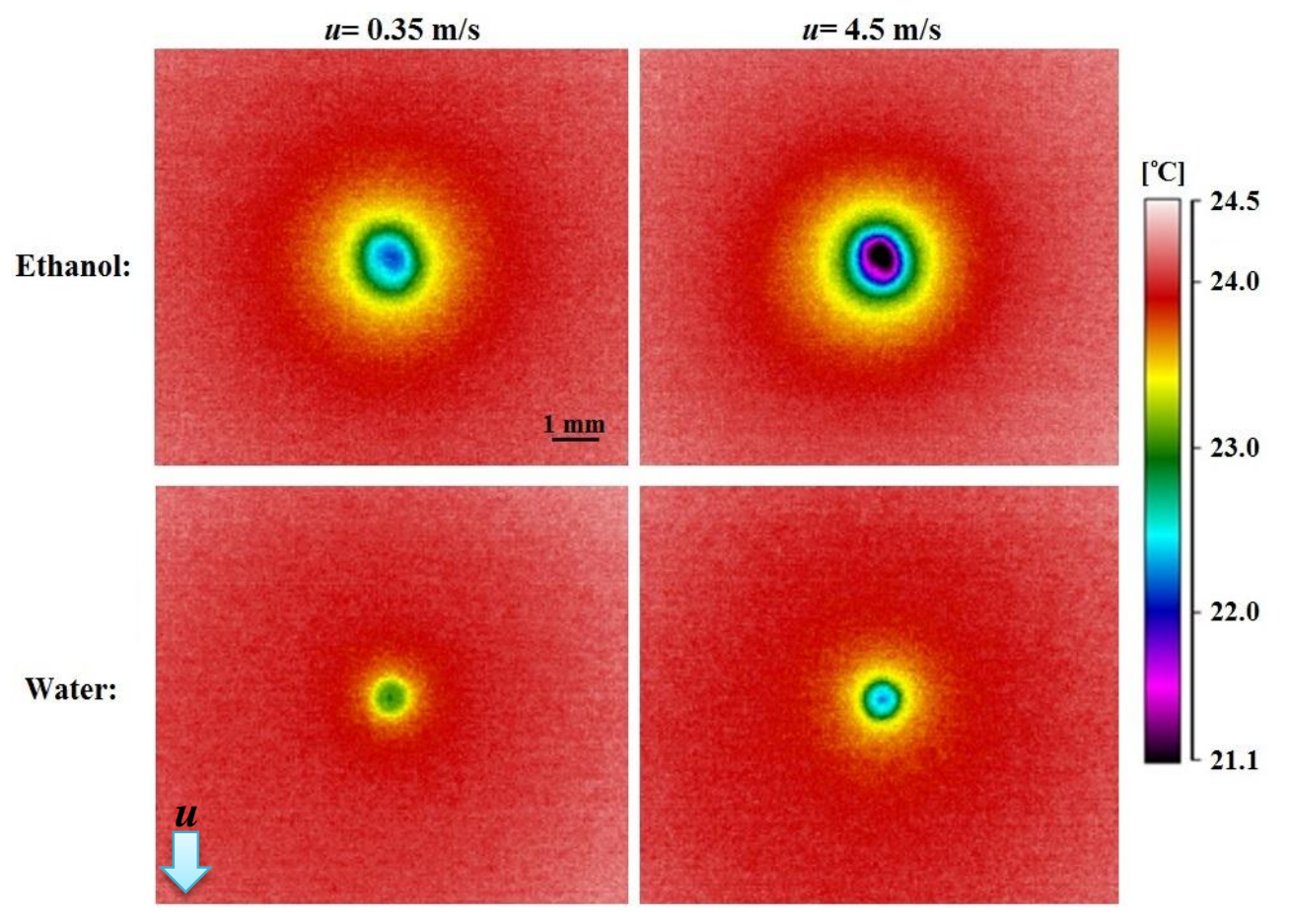

Figure 3. Infrared images of thermal field around an individual evaporating pore with diameter of $550 \mu \mathrm{m}$ drilled into the glass plate during ethanol and water evaporation for two different air velocities over the sample $\left(T_{a}=24 \pm 0.5{ }^{\circ} \mathrm{C}\right.$ and $\left.R H=40 \pm 2 \%\right)$. The arrow depicts air flow direction.

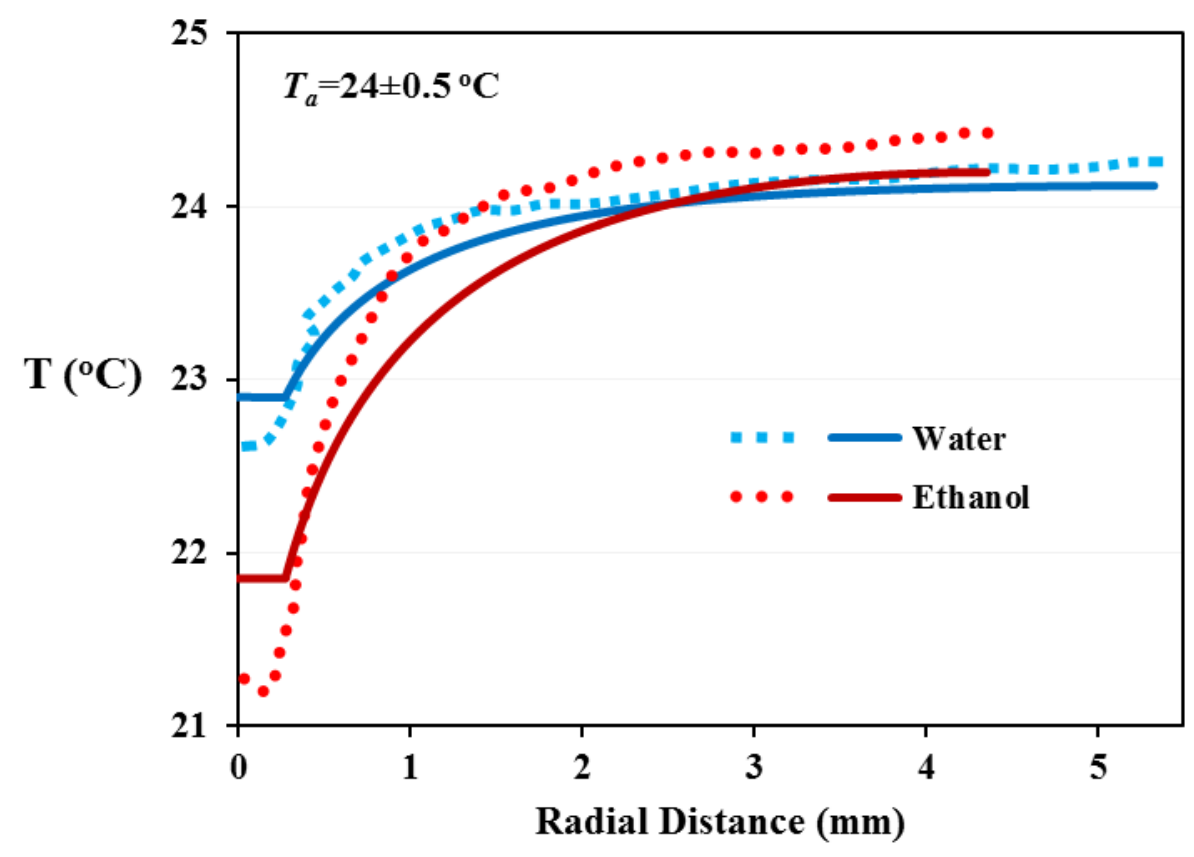

Figure 4. The radial temperature distribution around an individual evaporating pore with diameter of $550 \mu \mathrm{m}$; experimental results (symbols) obtained from microscopic infrared thermography and PCEB model predictions (solid lines) for air velocity of $4.5 \mathrm{~m} / \mathrm{s}$. 


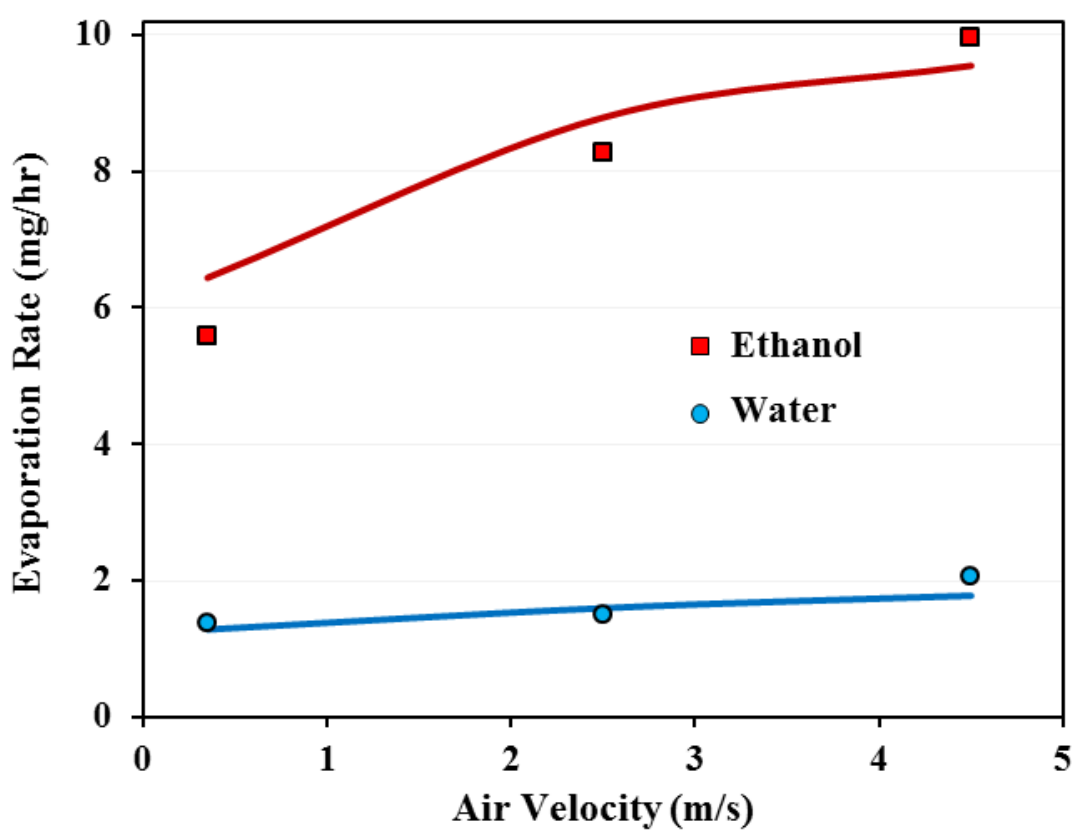

Figure 5. Model predictions (lines) and experimental results (symbols) of evaporation rate from a single evaporating pore $(\mathrm{d}=550 \mu \mathrm{m})$ during water and ethanol evaporation for three different air velocities as $0.35,2.5$, and $4.5 \mathrm{~m} / \mathrm{s}$ with $T_{a}=24 \pm 0.5^{\circ} \mathrm{C}$ and $R H=40 \pm 2 \%$.

\subsection{Effects of pore spacing on per-pore evaporation rate and surface temperature}

A critical aspect of PCEB model is the representation of changes in evaporation rates from individual pores as the surface dries (during stage 1) attributed to increased spacing between pores $[10,12]$. We evaluated measurements and predictions of evaporative fluxes from pore clusters with different spacing reflecting the variation of vapor flux from individual pores and its coupling with pore surface temperature to test this aspect of the model also in the context of energy balance. A cluster of 17 pores with same diameters $(550 \pm 10 \mu \mathrm{m})$ was drilled into the rough glass plates with three different spacing between adjacent pores as 2, 4, and $6 \mathrm{~mm}$. Evaporation experiments were conducted with air velocities of $0.35,2.5$, and $4.5 \mathrm{~m} / \mathrm{s}$ while recording the surface thermal patterns using infrared camera during ethanol evaporation. The high volatility of ethanol and resulting significant temperature depression on the surface of evaporating pores (Figure 3) provided resolved images that clearly capture differences between surface thermal signatures due to the variations in pore spacing and corresponding evaporation rates from pores in the cluster. 
The infrared images in Figure 6 depict thermal fields in pore clusters subjected to different air velocities and for different pore spacing during ethanol evaporation. For constant pore spacing, the increase in air velocity increases evaporative flux from the pore cluster and consequently results in larger temperature depression on the surface. Interestingly, pores located at the periphery of the cluster exhibit higher temperatures (despite lower diffusive resistance due to less neighboring pores and thus higher evaporative flux), probably due to higher lateral conductive energy flux from the surface. The center pore in the cluster with maximal number of neighboring pores exhibits the lowest surface temperature in the cluster due to thermal screening of peripheral pores.

Increasing pore spacing for similar number of pores and prescribed air velocity offers a means for systematic evaluation of the effects of pore spacing on surface thermal fields and evaporative losses. As seen in Figure 6, for constant boundary conditions, pore temperature increases with increasing pore spacing. PCEB model predictions of evaporation rates from cluster (expressed as evaporative mass loss, i.e., $\mathrm{g} / \mathrm{hr}$ ), and mean pore temperature (average of 17 pores) were compared with experimental results as illustrated in Figure 7. Model predictions were obtained based on spatial superposition of individual pores predictions. The results in Figure 7 demonstrate that evaporation rate from the cluster increases with increasing air velocity and with increased pore spacing for the same air velocity as expected from the theoretical study of Lehmann and Or [29]. Despite higher evaporation rates from the cluster with larger pore spacing implying higher evaporative flux from the surface of individual pores, higher pore temperature is observed in experiments and predicted by PCEB model. Increase of pore spacing increases surface of energy input into the unit cell (for a constant energy flux over the surface) and it occurs at a higher rate than the increase in per-pore evaporation rate due to the reduction of diffusive resistance (Appendix B). Consequently, conductive heat flux towards the evaporating pores increases yielding warmer pores that, in 
turn, contribute in the enhancement of vapor flux from individual pores by increasing vapor concentration gradient from pore surface across the mass boundary layer.
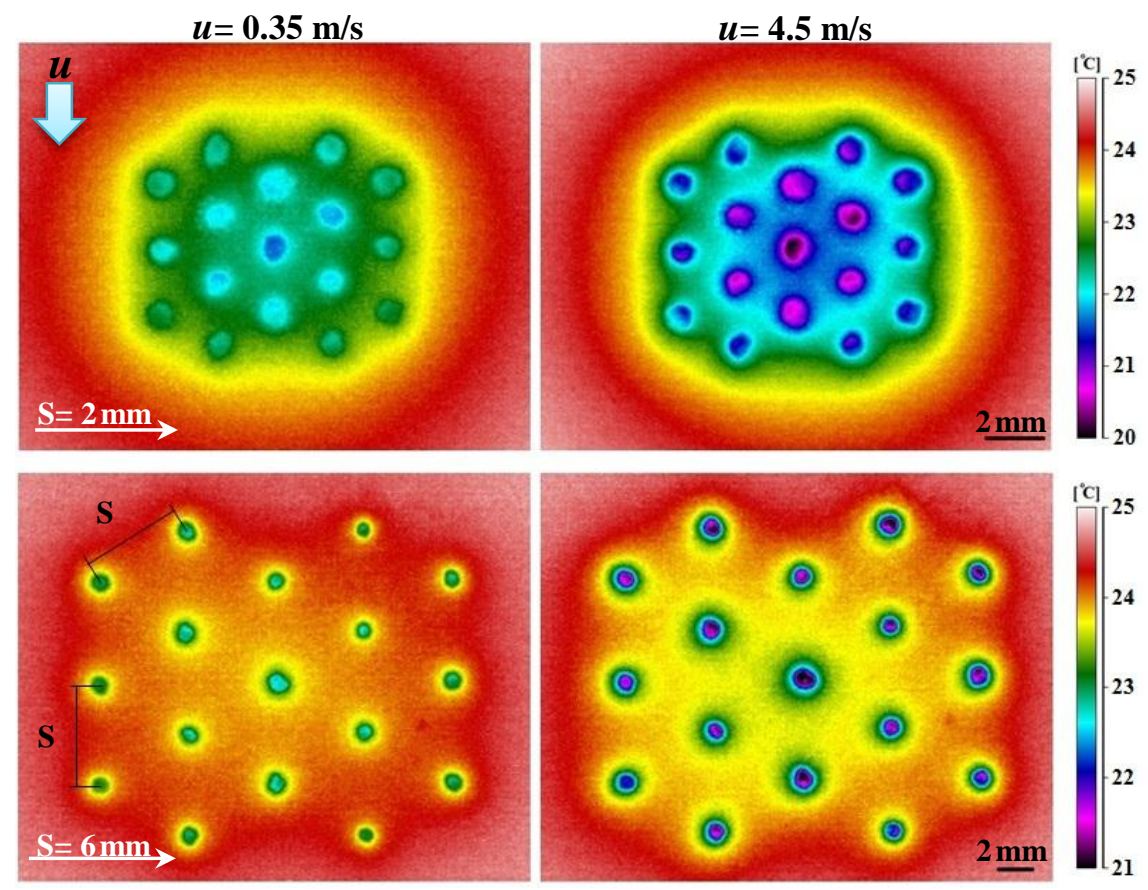

Figure 6. Infrared images of surface thermal fields forming around evaporating pore clusters with different pore spacing ( 2 and $6 \mathrm{~mm})$ and air velocities $(0.35$ and $4.5 \mathrm{~m} / \mathrm{s})$ during ethanol evaporation with $T_{a}=24 \pm 0.5{ }^{\circ} \mathrm{C}$. The clusters consist of 17 similar pores with diameters of $550 \pm 10 \mu \mathrm{m}$. Higher pore temperatures with increasing spacing are reflected in scale bars with different temperature ranges.

Figure 8 shows the evaporation ratio defined as mean per-pore evaporation rate from the cluster normalized by single pore evaporation rates for similar conditions (Figure 5) to illustrate the combined effects of pore spacing and air velocity on evaporation rates. The experimental results and model predictions confirm that per-pore evaporation rates increase with increasing pore spacing. As pore spacing exceeds the boundary layer thickness, the evaporative resistance (and thus evaporation rates) for pores in the cluster approaches that of individual pores; while for smaller spacing, interaction of vapor fields around individual surface pores increases diffusive resistance and suppresses evaporation rates [12,29]. This important aspect is evident in Figure 8 where pores in the clusters with different spacing evaporate at a rate similar to single pore by increasing air velocity (i.e., $u=4.5 \mathrm{~m} / \mathrm{s}$ ) that 
provides thinner mass boundary layer over the surface. The new aspect here is the role of energy input as reflected in surface temperature where the increased area of the unit cell dominates energetic aspects and yields warmer pores that, in turn, contribute in the enhancement of vapor diffusion with increasing spacing.
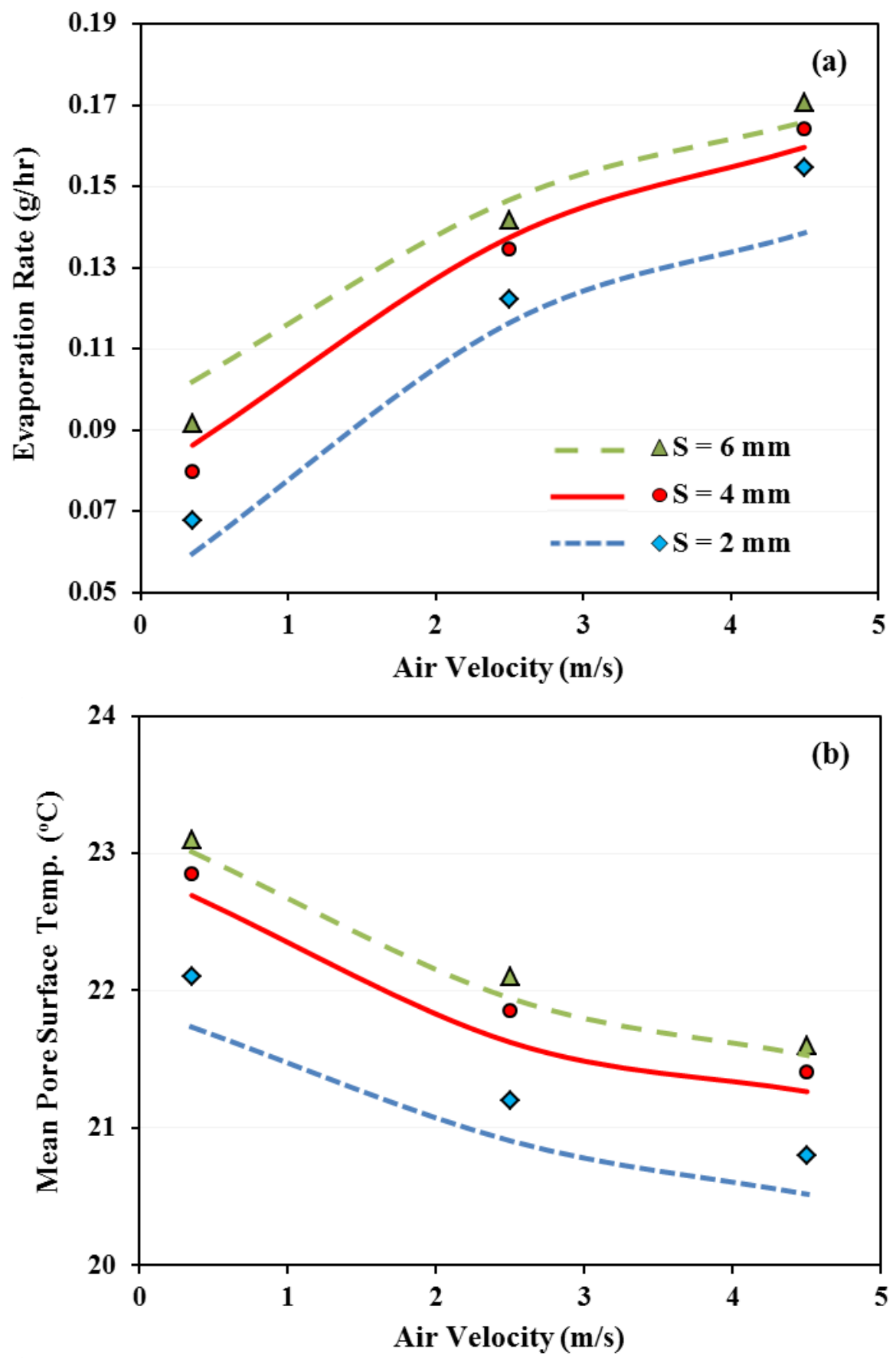

Figure 7. Model predictions (lines) and experimental results (symbols) of ethanol evaporation rate (a), and mean pore surface temperature (average of 17 pores) (b); model predictions obtained based on spatial superposition of individual pores predictions. 


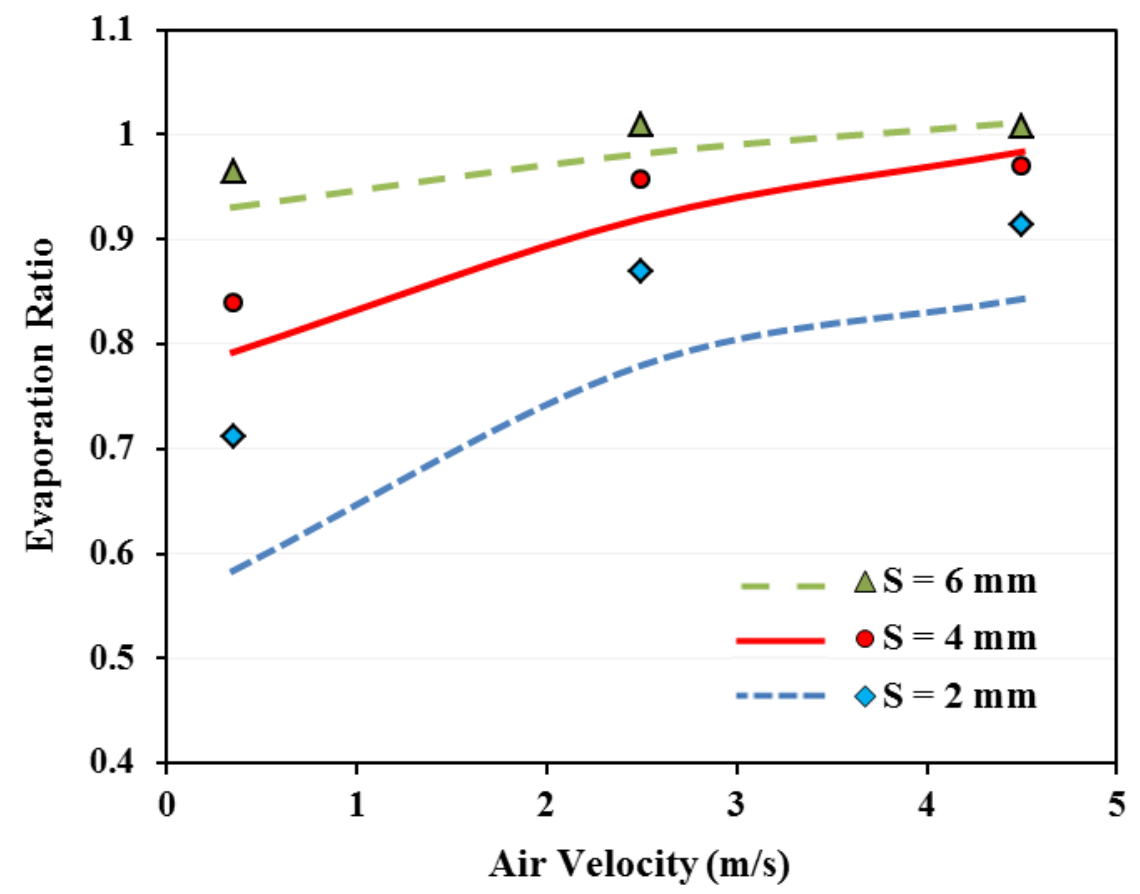

Figure 8. Model predictions (lines) and experimental results (symbols) of the evaporation ratio obtained by scaling the mean per-pore evaporation rate from the cluster with single pore evaporation results for ethanol shown in Figure 5.

To complete the picture, we conducted experiments using water as the evaporating liquid and compared these results with the ethanol tests to assess the roles of vapor diffusion process and latent heat of vaporization on evaporation rate and pore surface temperature. The comparisons between water and ethanol experimental results along with model predictions are shown in Figure 9. For the same air velocities the evaporation ratio defined as the ratio of water to ethanol evaporation rate $\left(E_{w} / E_{e}\right)$ increases by increasing spacing. This could be attributed to the lower Schmidt number of water $\left(S c_{w}=0.66\right)$ in comparison with ethanol $\left(S c_{e}=1.31\right)$ resulting in thicker mass boundary layer $\left(\delta_{m}\right)$ for water with the same air velocity over the surface (i.e., similar $\delta_{v}$ ). Hence, the evolution of vapor shells above individual surface pores with increasing spacing is more efficient for water evaporation and is also accompanied by a reduction of pore temperature ratio (i.e., $T_{w} / T_{e}$ ). The temperature depression on the surface of evaporating pores during ethanol evaporation was larger than for water due to the higher evaporative heat flux of ethanol that yields lower enhancement of vapor concentration 
gradient by increasing spacing. Considering different vapor concentration gradients and diffusion coefficients of water and ethanol, the water mass evaporation rate (i.e., $\left.E=D_{a} \Delta C / \delta_{m}\right)$ in the present experiments is almost $1 / 6$ of ethanol mass loss. Consequently, the higher latent heat of vaporization for water $\left(L_{w} / L_{e}=2.93\right.$, see Table A2) results in an evaporative heat flux (i.e., $L E$ ) that is about half of the ethanol and yields warmer pores (Figure 9).

\subsection{Effects of shortwave radiation on the temperature of evaporating surfaces}

The crucial role of shortwave radiation on pore-scale evaporation dynamics was studied experimentally by contrasting thermal fields and evaporation rates in the presentence and absence of shortwave radiation flux over the surface (i.e., $R_{S}=0 \mathrm{~W} / \mathrm{m}^{2}$ and $R_{S}=300 \mathrm{~W} / \mathrm{m}^{2}$ ). The resulting IR images for a cluster with spacing of $6 \mathrm{~mm}$ during water evaporation are depicted in Figure 10. The results show that increasing shortwave radiation affects mean surface temperature and dynamics of thermal fields forming around surface pores. The measured radial temperature distributions around the center pore in the cluster were compared with PCEB model predictions in the presence and absence of radiation as depicted in Figure 11. The resulting temperature field around evaporating pores in the presence of shortwave radiation shows sharper temperature gradients indicative of higher conductive heat flux towards the pore relative to no radiation case. The different radiation regimes were expected to affect mean surface temperature as also seen in model predictions in Figure 12 (focusing on the hexagonal areas), with significantly higher surface temperature for irradiated surfaces. Consequently, the vapor concentration over evaporating pores increases and results in higher evaporation rates from the radiated pore cluster relative to the surface with no shortwave radiation. 

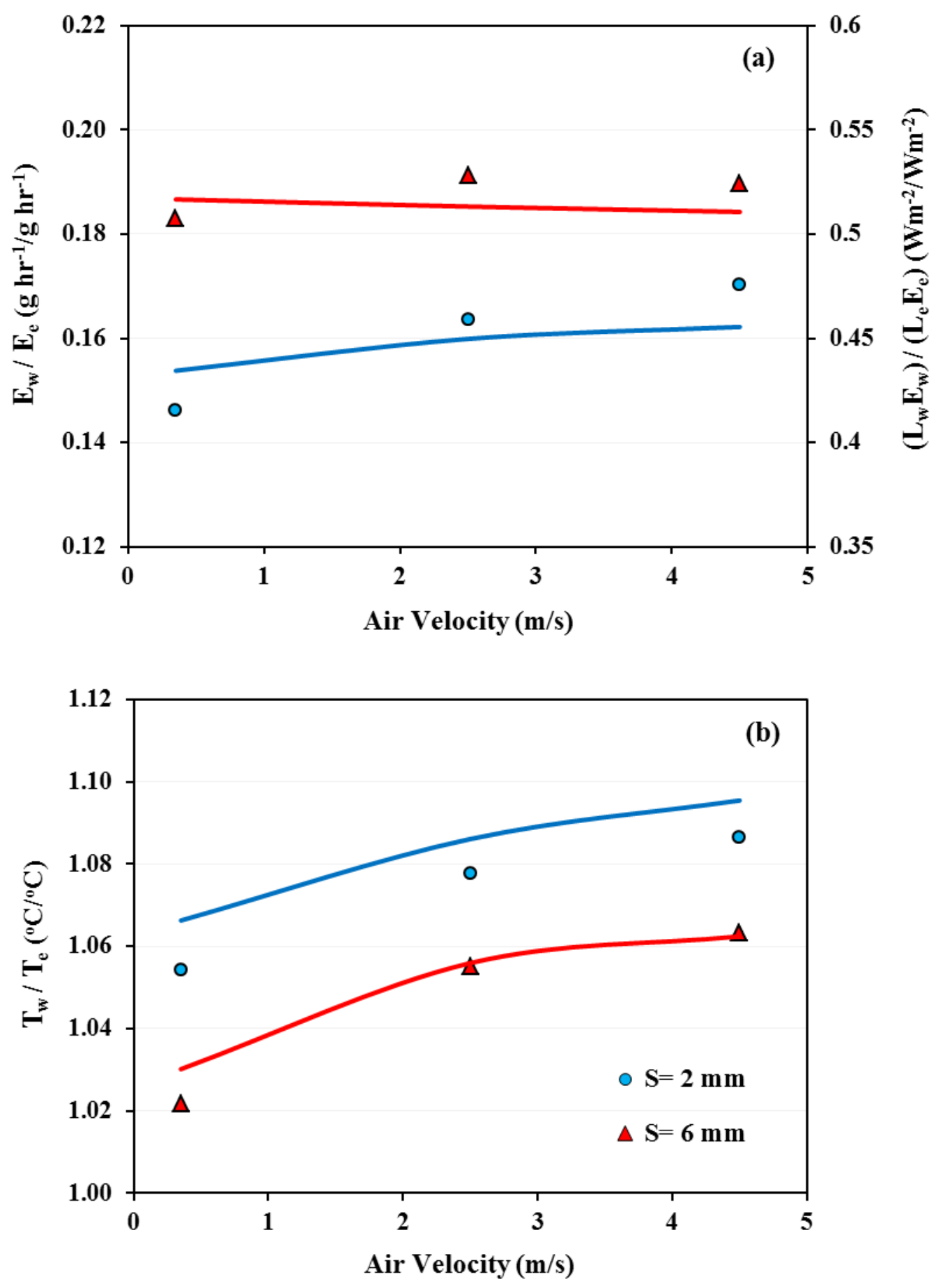

Figure 9. Experimental results (symbols) and model predictions (lines) of evaporative mass loss ratio of water to ethanol $\left(E_{w} / E_{e}\right)$, and the equivalent evaporative heat flux ratio of these two liquids ( $L_{w} E_{w} / L_{e} E_{e}$ ) (a) and the resulting pore surface temperature ratio $\left(T_{w} / T_{e}\right)$ (b) vs. air velocity for pore spacing of 2 and $6 \mathrm{~mm}$. 

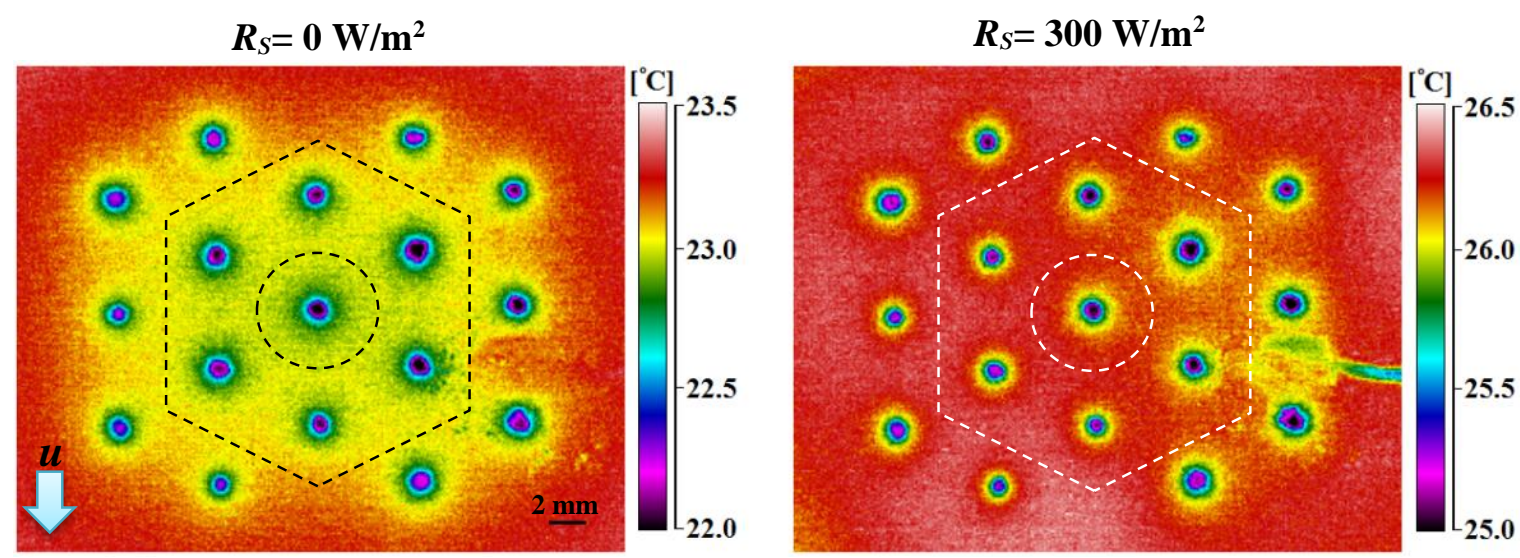

Figure 10. IR images of thermal fields on the surface of pore cluster with spacing of $6 \mathrm{~mm}$ during water evaporation for shortwave radiation fluxes of 0 and $300 \mathrm{~W} / \mathrm{m}^{2}$, air velocity of $4.5 \mathrm{~m} / \mathrm{s}$, and $T_{a}=24 \pm 0.5^{\circ} \mathrm{C}$. The expected increase in surface temperature with increasing radiation is evident in temperature scale bars.

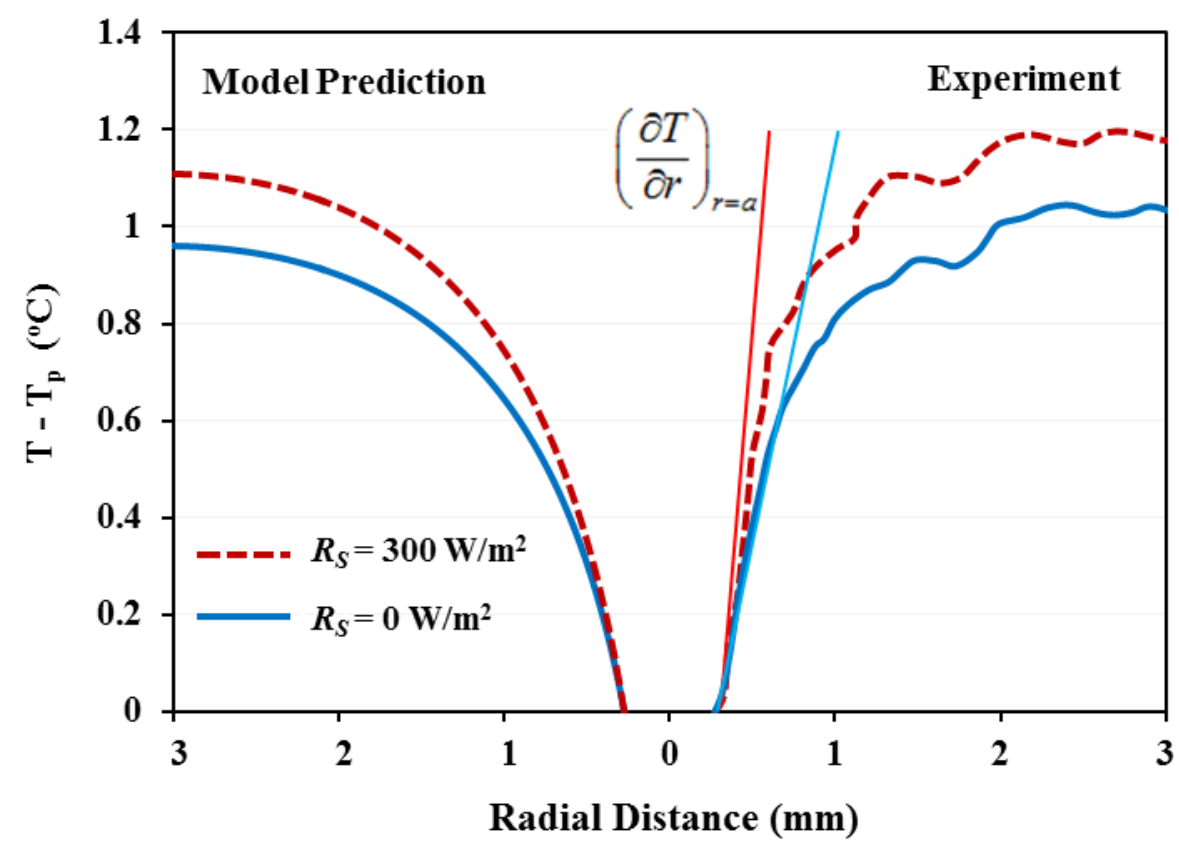

Figure 11. Model predictions (left) and experimental results (right) of temperature distribution around the center pore of the cluster (marked in Figure 10) with respect to the pore temperature $\left(T_{p}\right)$ measured as $22.1{ }^{\circ} \mathrm{C}$ and $25.2{ }^{\circ} \mathrm{C}$ for $R_{S}=0 \mathrm{~W} / \mathrm{m}^{2}$ and $R_{S}=300 \mathrm{~W} / \mathrm{m}^{2}$, respectively. The inclined thin lines represent thermal gradients at pore wall $(r=0.275 \mathrm{~mm})$. 

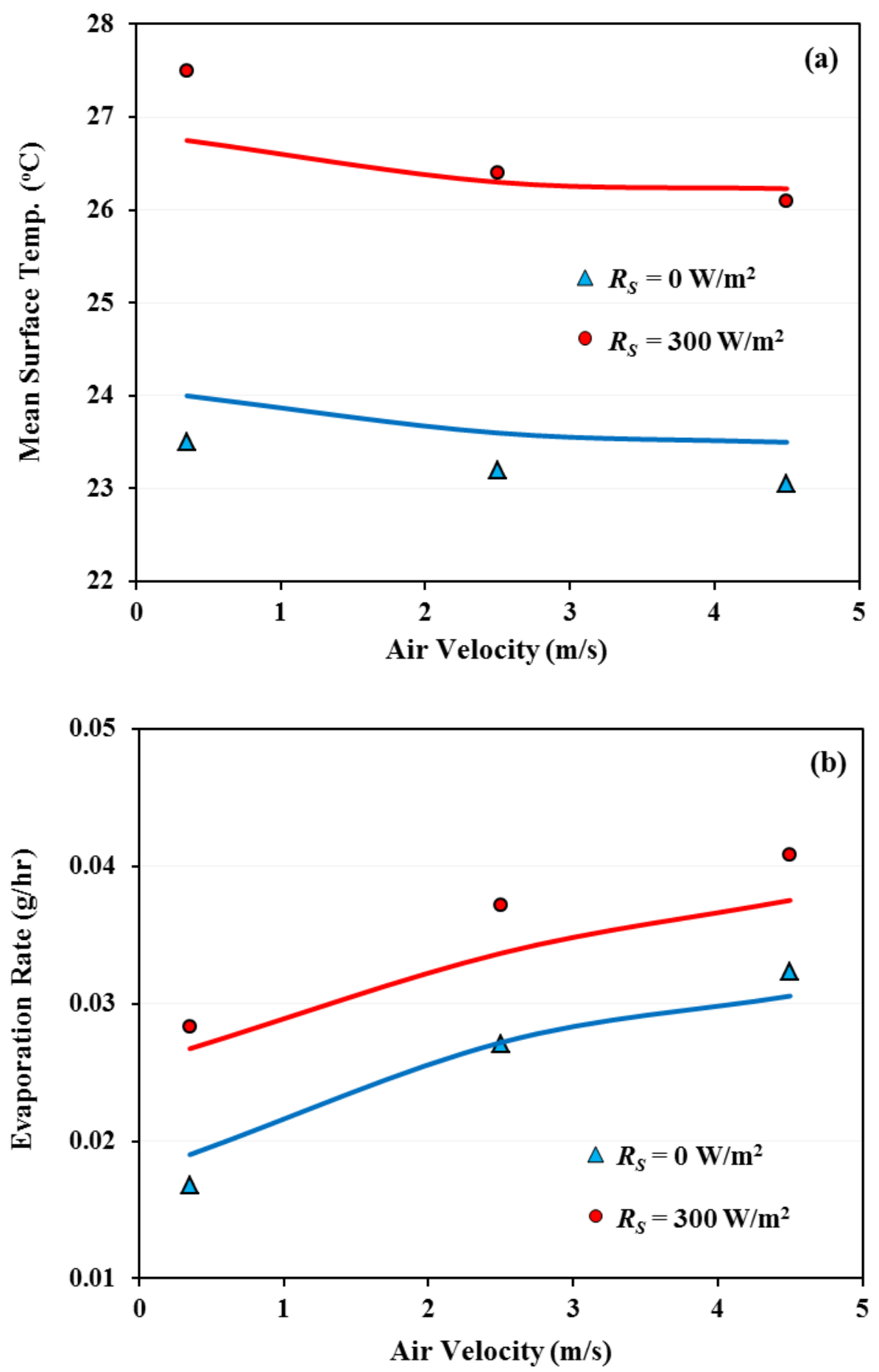

Figure 12. Model predictions (lines) and experimental results (symbols) of mean surface temperature (marked area by hexagons in Figure 10) (a), and evaporation rate from the cluster of water filled pores (b) vs. air velocity for different radiative fluxes over the sample.

\subsection{Dynamics of pore-scale thermal adjustment}

The dynamic nature of surface drying raises the question of how quickly a surface adjusts thermally to pore emptying and whether the PCEB solution strategy based on steady state 
steps is justified. This aspect has been investigated experimentally using microscopic infrared thermography. A pore in a cluster was blocked by a wire (a piece of soldering wire) and the evaporating surface was allowed to reach thermal and evaporative steady state before removing the wire and observing the thermal field adjustment around the previously blocked pore as it resumes evaporation. Figure 13 depicts a typical temporal evolution of the thermal field around the center pore of cluster for dense $(S=2 \mathrm{~mm})$ and sparse $(S=4 \mathrm{~mm})$ pore spacing during ethanol evaporation. The IR images at $\mathrm{t}=0 \mathrm{~s}$ depict temperature distribution around the blocked pore. The sequence of surface IR images demonstrates that surface temperature attains a steady state within a short time (less than $2 \mathrm{~s}$ ) and the equilibration occurs faster in dense pore spacing (higher surface liquid content). These experimental results support the assumption of steady state conditions in application of PCEB model for stepwise drying of a surface as a function of surface liquid content (through a series of steady state steps).
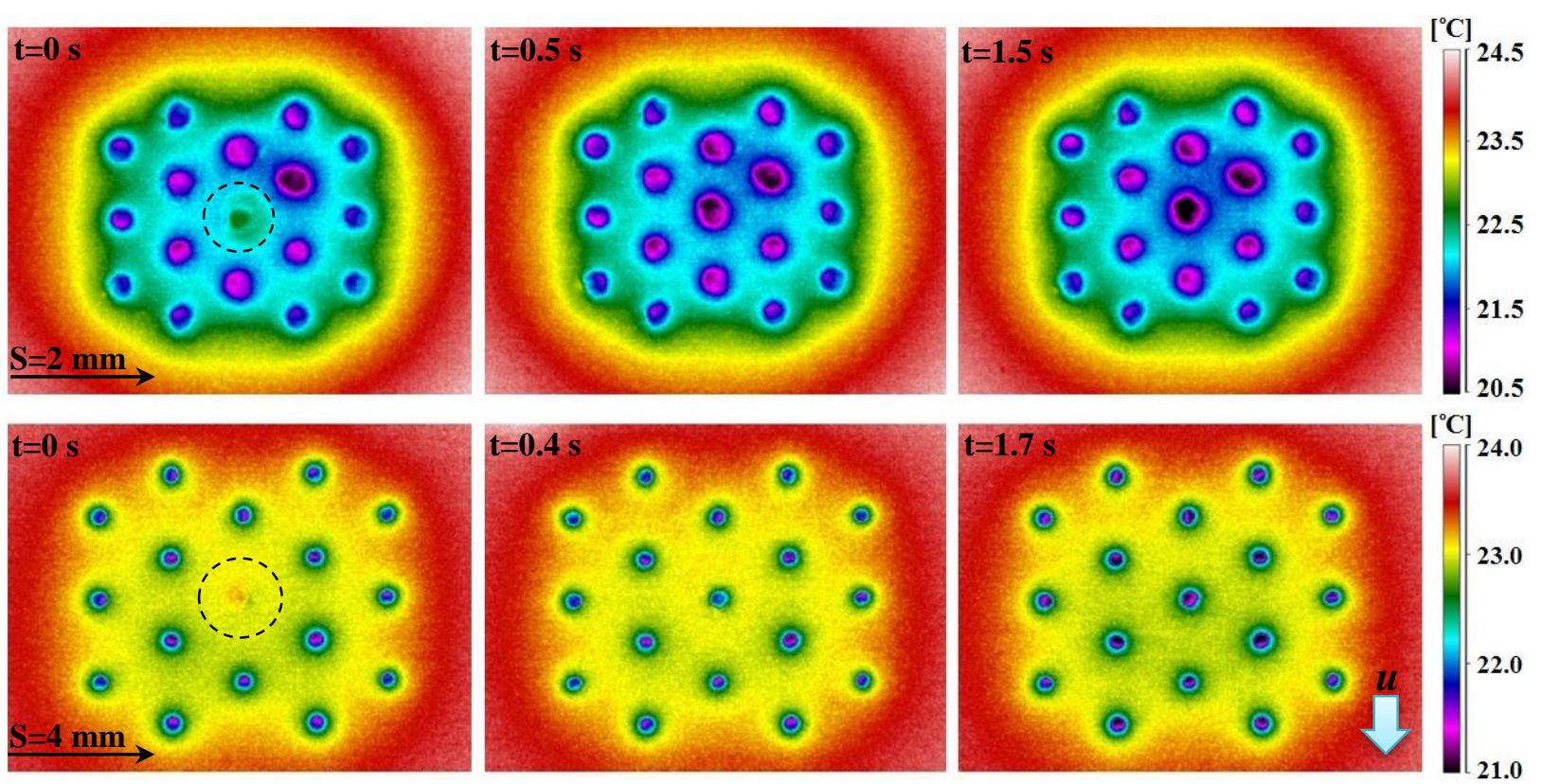

Figure 13. Infrared images of thermal adjustment dynamics around individual pores in pore clusters with pore spacing of 2 and $4 \mathrm{~mm}$, constant air velocity of $2.5 \mathrm{~m} / \mathrm{s}$ over the surface and $R_{S}=0 \mathrm{~W} / \mathrm{m}^{2}$. Blockage of the center pore is removed at $\mathrm{t}=0 \mathrm{~s}$ marking the onset of evaporation from that pore and subsequent adjustment of the thermal field. 


\subsection{Evaporative pore emptying and thermal adjustments over a porous surface}

The mechanistic picture for evaporative drying of porous surfaces in which invasion of surface pores affects the average spacing between remaining wet pores and modifies energy partitioning has not been tested at pore-scale. Figure 14 depicts optical and thermal images of a porous surface made up of a monolayer of glass beads with diameter ranging from 1.6 to 2.4 $\mathrm{mm}$. The surface was initially fully saturated with ethanol and sequential emptying of surface pores during evaporation and concurrent adjustments of thermal fields were recorded using an optical microscope and highly resolved IR thermography. Comparing optical images at different time steps demonstrates that sequential invasion of surface pores is governed by capillary processes determined by pore geometry from larger to smaller pores. It is relatively simple to predict the pore emptying sequence at a surface of such coarse glass beads sample based on the size of inscribed circles within surface pores that represents the air-entry pressure at pore invasion for all surface pores. The agreement between predicted pore emptying sequence based on pore geometry and observed pore emptying from optical imaging was very good (Figure 15) suggesting a means for linking predictable pore emptying sequences with variations in mean surface temperature (Appendix C).

As seen in thermal images of Figure 14, the emptying of surface pores is accompanied by rapid temperature adjustment in adjacent glass beads and neighboring "wet" pores. The intercepted energy flux at the surface of drying sample that is not used by evaporation from empty surface pores primarily increases the temperature of adjacent glass beads and modifies the conductive heat fluxes around the neighboring wet pores. Accordingly, an evaporating pore that is surrounded by invaded pores has higher temperature in comparison with evaporating pores with wet neighboring pores (see dashed circles in Figure 14). This porescale (nearly instantaneous) adjustment of temperature distribution induced by evaporative pore emptying confirms the coupling between surface temperature and evaporative flux 
during drying of porous surfaces that is in the basis of PCEB model. It also supports the proposed quasi steady state solution of PCEB as the dynamics are very rapid.

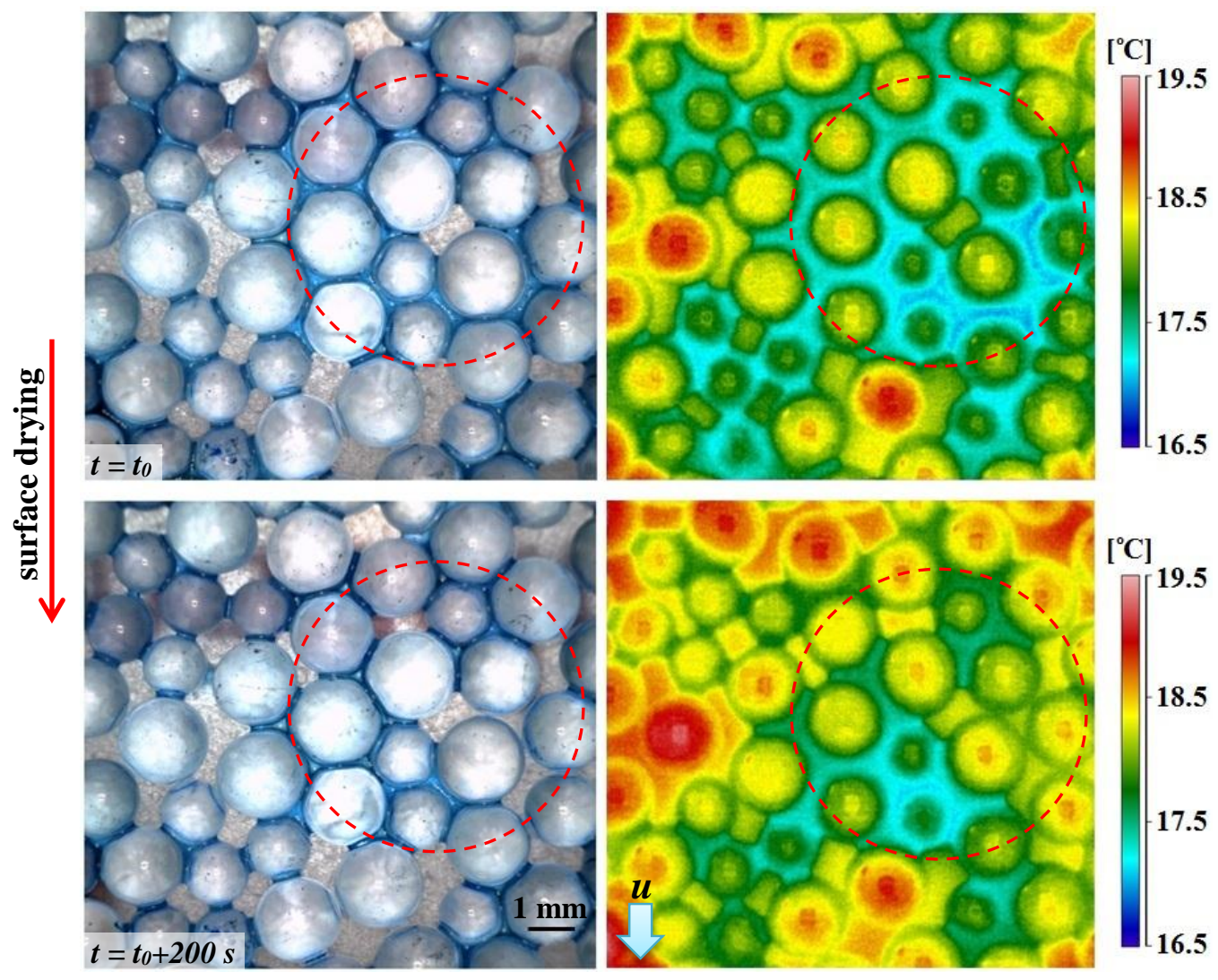

Figure 14. Optical (left) and thermal (right) images of glass beads surface during ethanol evaporation with air velocity of $4 \mathrm{~m} / \mathrm{s}, R_{S}=0 \mathrm{~W} / \mathrm{m}^{2}$, and $T_{a}=24.5^{\circ} \mathrm{C}$. Significant temperature depression due to the ethanol vaporization marks evaporating pores while pore emptying (circles) is accompanied by rapid temperature increase of adjacent glass beads and neighboring wet pores.

The evolution of evaporative flux from the glass beads sample (measured by a digital balance) and the corresponding mean surface temperature (monitored by IR imager) along with PCEB predictions are presented in Figure 16. Model predictions were obtained based on the unit cell representation of surface drying where reduction of surface liquid content is attributed to the increase of dry region around the representative pore whose diameter was estimated as $1 / 3$ of the mean particle size [30]. The results demonstrate that reduction of evaporative mass loss during surface drying (less evaporating sources) yields concurrent increase of mean surface temperature [15]. The agreement between model predictions and experimental results 
highlights potential applicability of PCEB to represent mean surface temperature and evaporative fluxes over drying porous media.
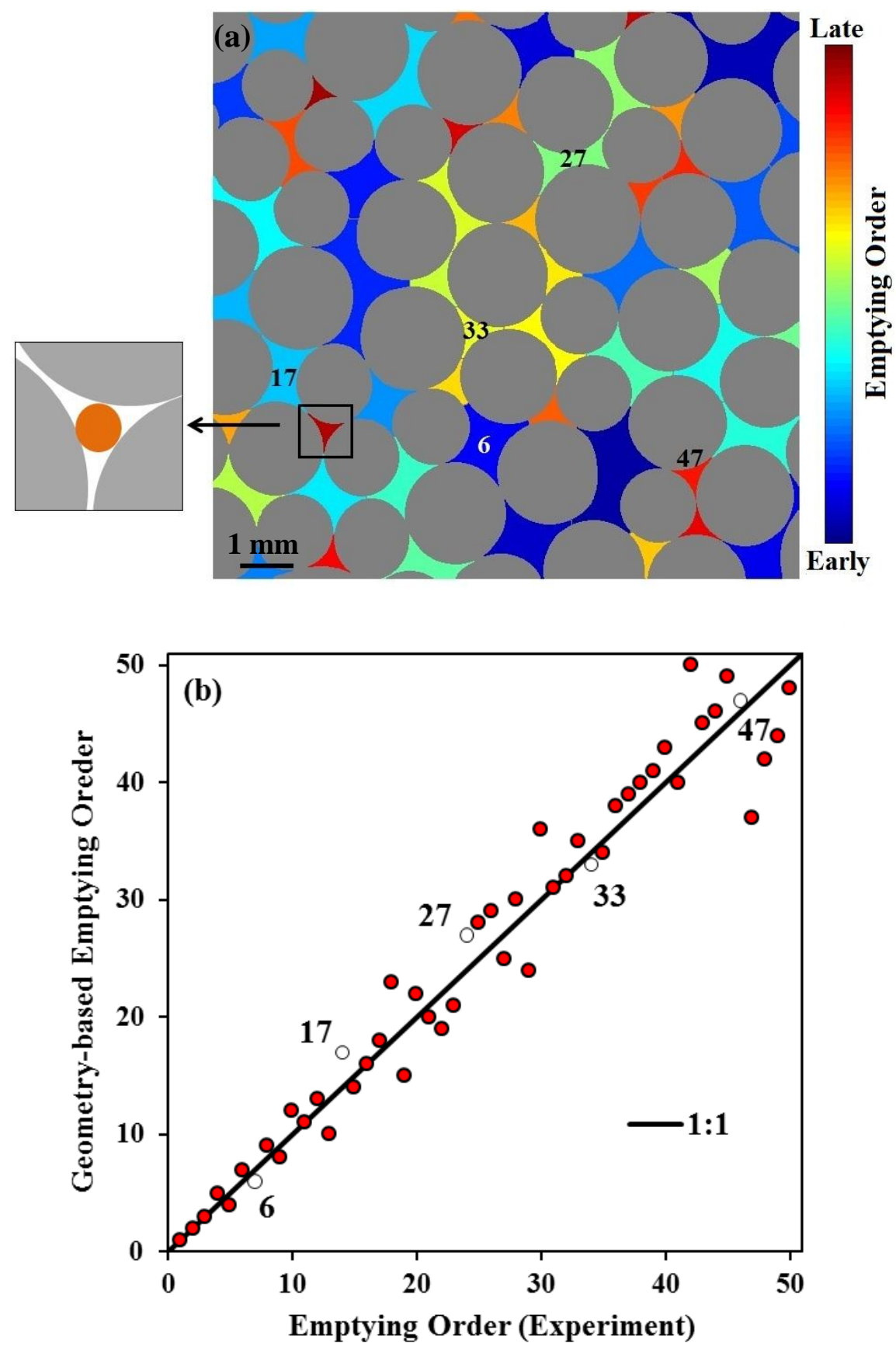

Figure 15. Pore emptying sequence during ethanol evaporation based on the size of inscribed circles in surface pores of monolayer glass beads sample with grain size ranging from 1.6 to $2.4 \mathrm{~mm}$ (a); comparison between emptying order based on pore geometry and observed pore emptying sequence (b). 


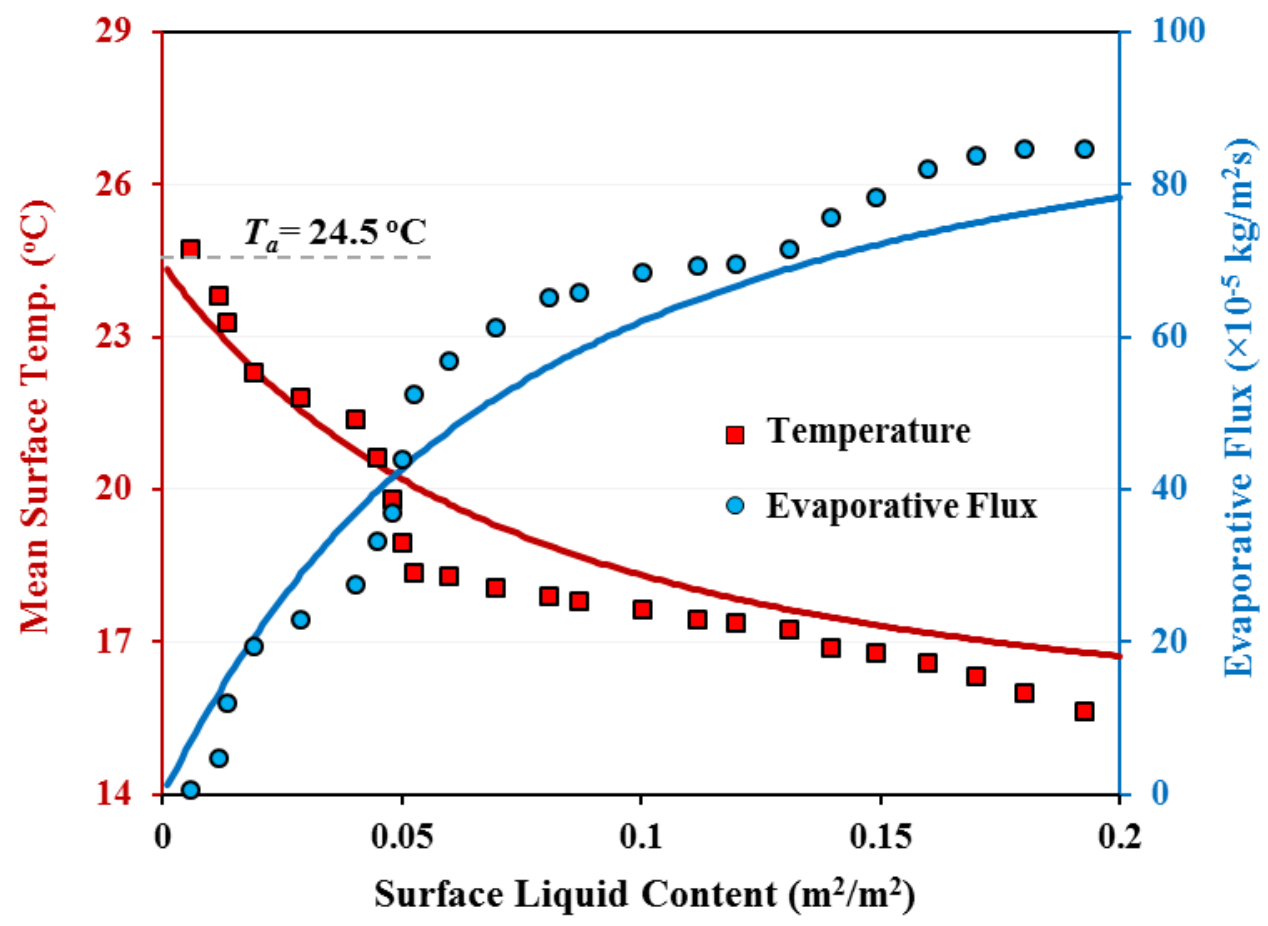

Figure 16. Model predictions (lines) and experimental results (symbols) of mean evaporative flux and mean surface temperature during drying of the glass beads sample in Figure 14. For the comparison with model predictions, variations in surface liquid content during drying experiment were extracted from the sequence of optical images (estimating the wet area loss with pore invasion).

\section{Summary and conclusions}

The Pore-scale Coupled Energy Balance (PCEB) model of Aminzadeh and Or [22] enables analytical description of the coupling between surface temperature and evaporative flux from drying porous surfaces formulated as a collection of evaporating pores [12]. The derivation of an analytical solution that enables prediction of energy partitioning over the surface relies on several untested assumptions. We aimed to test some of the building blocks in the basis of the PCEB model, specifically, the following questions are addressed: (1) are the pore-centered description of drying porous surfaces and the lateral exchanges assumed in the PCEB justified and they resemble pore-scale (idealized) experimental conditions? (2) is the pore-scale description upscalable to represent surfaces with different pore patterns and liquid contents? 
and (3) can the pore-scale representation of evaporating surface capture dynamics of energy partitioning over drying surfaces?

Microscopic infrared thermography and well defined porous surfaces were employed to measure details of thermal fields around individual surface pores and their interactions. Evaporation rates and temperature distribution measured around pores and pore clusters drilled in rough glass enabled systematic evaluation of PCEB model predictions. Good agreement was obtained for the thermal field around a single evaporating pore based on highly resolved infrared images. The model was then employed to investigate the effect of pore spacing on the enhancement of diffusive mass loss from individual surface pores for sparse and dense pore spacing based on spatial superposition of individual pores predictions $[11,29]$. Both PCEB and experimental results showed that increase of pore spacing increases per-pore evaporative flux through the evolution of diffusive vapor shells and pore-scale adjustment of energy partitioning on the surface. Model prediction of temperature distribution around surface pores in the presence of radiative flux over the sample was in good agreement with experimental results demonstrating that existence of shortwave radiation results in sharper thermal gradients around surface pores and enhances evaporative mass loss by increasing mean surface temperature.

The drying dynamics of a monolayer of glass beads was observed using optical and thermal imaging to address the link between pore emptying sequence during evaporative drying and adjustment of thermal fields around surface pores. The pore emptying sequence obtained from optical imaging was in excellent agreement with the emptying order predicted from pore geometry (and capillary forces) confirming that pore size distribution governs the order of pore invasion during evaporative surface drying. Additionally, surface thermal signatures recorded by thermal camera showed that pore emptying process is accompanied by rapid 
temperature increase of adjacent glass beads that, in turn, adjusts lateral conductive heat fluxes towards the neighboring wet pores.

Results of this study confirm PCEB model's capability to predict energy partitioning during drying of porous surfaces and highlight the crucial role of pore-scale thermal adjustments in description of coupled heat and mass transfer processes when porous surfaces gradually dry.

\section{Acknowledgments}

The authors gratefully acknowledge funding by the Swiss National Science Foundation (200021-113442) and generous assistance of Daniel Breitenstein and Hans Wunderli in experimental aspects of the study. The manuscript greatly benefited from insightful discussions with Dr. Peter Lehmann (ETH Zurich).

\section{Appendix A: A closed-form expression for predicting surface temperature}

The closed-form of surface temperature distribution on the surface of unit cell (Eq. 3) is obtained based on linearization of saturated vapor concentration curve and quantification of conductive heat flux across the pore wall [22].

Assuming liquid vapor as an ideal gas, saturated vapor concentration at the surface of evaporating pores is obtained as:

$$
C_{s}=\frac{M P_{s}}{R T_{l}}
$$

where $M$ is the molar mass of evaporating liquid $[\mathrm{kg} / \mathrm{mol}], R$ is the molar gas constant $[8.314 \mathrm{~J} / \mathrm{mol} \mathrm{K}], T_{l}$ is the mean liquid surface temperature $[\mathrm{K}]$ and $P_{s}$ is the saturated vapor pressure $[\mathrm{Pa}]$ that can be quantified based on the Antoine equation as a function of liquid temperature $\left(T_{l}\right.$ in degree of Celsius $\left.\left[{ }^{\circ} \mathrm{C}\right]\right)[31]$ :

$$
P_{s}=133.32 \times 10^{A-\frac{B}{C+T_{l}}}
$$


in which $A, B$ and $C$ are Antoine equation parameters (Table A1). Physical properties of water and ethanol have been presented in Table A2.

Based on Eqs. (A1) and (A2), $C_{s}$ is a non-linear function of temperature. Hence, we linearize $C_{s}$ for a finite range around air temperature $( \pm 10 \mathrm{~K})$ that leads to the following expression for saturated vapor concentration at pore surface as a function of pore temperature:

$$
C_{s}=a^{\prime} T_{l}+b^{\prime}
$$

where $a^{\prime}$ and $b^{\prime}$ are linearization constants obtained as $a^{\prime}=0.007 \mathrm{~kg} / \mathrm{m}^{3} \mathrm{~K}$ and $b^{\prime}=-1.937$ $\mathrm{kg} / \mathrm{m}^{3}$ for ethanol, and $a^{\prime}=0.001125 \mathrm{~kg} / \mathrm{m}^{3} \mathrm{~K}$ and $b^{\prime}=-0.3116 \mathrm{~kg} / \mathrm{m}^{3}$ for water in evaporation experiments of this study with air temperature around $24{ }^{\circ} \mathrm{C}(297.15 \mathrm{~K})$. Accordingly, insertion of Eq. (A3) into the pore liquid energy balance (Eq. 4) near the surface $(\Delta h)$ leads to the following expression for conductive heat flux across the pore wall:

$$
q_{\text {cond }}=\frac{a}{2 \Delta h}\left(C_{2} T_{l}+C_{3}\right)
$$

where $C_{2}$ and $C_{3}$ are defined as:

$$
\begin{gathered}
C_{2}=C_{1} a^{\prime}+h_{a}+4 \sigma \varepsilon_{l} T_{a}^{3}+\frac{k}{\Delta H} \\
C_{3}=C_{1} b^{\prime}-C_{1} C_{a}-R_{S}-h_{a} T_{a}-\left(3 \varepsilon_{l}+\varepsilon_{a}\right) \sigma T_{a}^{4}-\frac{k}{\Delta H} T_{s}
\end{gathered}
$$

in which $\varepsilon_{l}$ is the liquid emissivity and parameter $C_{1}$ represents the mass transfer coefficient (Eq. 6). To facilitate quantification of mean liquid temperature at pore surface we assume that pore surface temperature is simply the temperature of unit cell at $r=a\left(T(a)=T_{l}\right)$. Hence, $T_{l}$ is quantified using surface temperature equation (Eq. 3) as: 


$$
T_{l}=\frac{q_{\text {cond }}}{k \sqrt{\psi}} \times \frac{\left[\frac{K_{1}(\sqrt{\psi} b)}{I_{1}(\sqrt{\psi} b)} I_{0}(\sqrt{\psi} a)+K_{0}(\sqrt{\psi} a)\right]}{\left[\frac{K_{1}(\sqrt{\psi} b)}{I_{1}(\sqrt{\psi} b)} I_{1}(\sqrt{\psi} a)-K_{1}(\sqrt{\psi} a)\right]}+\varphi \quad a \leq r \leq b
$$

Introducing variable $C_{4}$, the liquid temperature is written as:

$$
\begin{gathered}
C_{4}=\frac{1}{k \sqrt{\psi}} \times \frac{\left[\frac{K_{1}(\sqrt{\psi} b)}{I_{1}(\sqrt{\psi} b)} I_{0}(\sqrt{\psi} a)+K_{0}(\sqrt{\psi} a)\right]}{\left[\frac{K_{1}(\sqrt{\psi} b)}{I_{1}(\sqrt{\psi} b)} I_{1}(\sqrt{\psi} a)-K_{1}(\sqrt{\psi} a)\right]} \\
T_{l}=C_{4} \times q_{\text {cond }}+\varphi
\end{gathered}
$$

inserting $q_{\text {cond }}$ from Eq. (A4):

$$
T_{l}=\frac{a}{2 \Delta h} C_{4}\left(C_{2} T_{l}+C_{3}\right)+\varphi
$$

finally, mean liquid temperature is found by rearrangement of Eq. (A10) as:

$$
T_{l}=\frac{C_{4} C_{3} \frac{a}{2 \Delta h}+\varphi}{1-C_{4} C_{2} \frac{a}{2 \Delta h}}
$$

Determination of pore surface temperature provides closed-form of temperature distribution equation (Eq. 3) on the surface of unit cell by quantification of conductive heat flux across the pore wall using Eq. (A4).

Table A1. Antoine equation parameters for water and ethanol.

\begin{tabular}{|c|c|c|c|c|}
\hline Substance & A & B & C & Range of Validity \\
\hline Water & 8.07131 & 1730.63 & 233.426 & 1 to $100^{\circ} \mathrm{C}$ \\
\hline Ethanol & 8.20417 & 1642.89 & 230.300 & -57 to $80^{\circ} \mathrm{C}$ \\
\hline
\end{tabular}


Table A2. Physical properties of water and ethanol at $25^{\circ} \mathrm{C}$.

\begin{tabular}{|c|c|c|c|c|c|c|}
\hline Substance & $L(\mathrm{~kJ} / \mathrm{kg})$ & $D_{a}\left(\mathrm{~m}^{2} / \mathrm{s}\right)$ & $\rho\left(\mathrm{kg} / \mathrm{m}^{3}\right)$ & $M(\mathrm{~kg} / \mathrm{mol})$ & $\varepsilon_{l}$ & $S c$ \\
\hline Water & 2450 & $2.5 \times 10^{-5}$ & 998 & 0.018 & 0.95 & 0.66 \\
\hline Ethanol & 837 & $1.1 \times 10^{-5}$ & 786 & 0.046 & 0.95 & 1.31 \\
\hline
\end{tabular}

\section{Appendix B: Pore-scale adjustments of surface energy balance closure}

The increased dry area around evaporating pores on the surface during gradual surface drying is accompanied by an incremental increase in energy input intercepted by the expanding unit cell surface. The resulting increase in energy available for evaporation occurs while increased spacing decreases diffusive vapor resistance from the pore and increases evaporation capacity. We have investigated the potential effects of these two processes (increased available energy for evaporation and variations in diffusive resistance) that are in the core of the often observed constant surface temperature during stage 1 evaporation (see Aminzadeh and Or [15]) by increasing unit cell dry area around individual evaporating pores. In other words, we seek to find the pore spacing (water content) at which the input energy exceeds the evaporation capacity of the pore and thus inability to maintain a constant surface temperature by evaporative cooling.

To address these important aspects during surface drying, the change in energy budget of unit cell $\left(E_{b}\right)$ with incremental increase of the unit cell radius was investigated:

$$
\frac{\partial E_{b}}{\partial r}=\frac{2}{r}\left(\left(R_{n}-G-H\right) \times \pi r^{2}-L E \times \pi r^{2}\right)
$$

where $R_{n}$ is the net radiation flux, $H$ is the convective heat exchange between surface and air, $G$ is the vertical conductive heat flux and $L E$ is the evaporative flux. Figure B1 depicts variations of energy input $\left(\left(R_{n}-G-H\right) \times \pi r^{2}\right)$ and evaporative cooling capacity $\left(L E \times \pi r^{2}\right)$ with increase of unit cell's radius assuming constant surface temperature. As depicted, the increase of surface temperature decreases available energy in the unit cell thorough heat 
losses (e.g., outgoing long wave radiation) while evaporation capacity increases nonlinearly due to increased pore spacing [12]. For a prescribed constant surface temperature, the intersection of the input energy and the evaporation capacity curves represents a specific radius where surface energy balance occurs. This specific radius $\left(r^{*}\right)$ is found through the nuanced representation of surface energy balance equation based on the pore-scale diffusive vapor flux as:

$$
\left(R_{n}-G-H\right)-\frac{1}{1+\frac{r}{\delta_{m} \sqrt{\pi}}\left(\frac{\sqrt{\pi}}{2 a} r-1\right)} \frac{D_{a} L \Delta C}{\delta_{m}}=0
$$

that yields the following expression for $r^{*}$ :

$$
r^{*}=\frac{a}{\sqrt{\pi}}+\sqrt{\frac{a^{2}}{\pi}+2 a\left(\frac{D_{a} L \Delta C}{\left(R_{n}-G-H\right)}-\delta_{m}\right)}
$$

As seen in Figure B1, for smaller radii than $r^{*}$ the evaporation capacity exceeds the available energy meaning that $\partial E_{b} / \partial r<0$ and evaporation is constrained by input energy; thus surface temperature adjusts to meet energy balance closure (as enforced by PCEB model). For larger unit cell radii the available energy exceeds evaporation capacity and the surface temperature for energy balance closure is higher suggesting surface warming and a shift in energy partitioning between latent and sensible heat fluxes with surface drying. Such adjustment in surface temperature is sharper at low surface water contents marking transition to stage 2 evaporation with concurrent decrease in evaporative flux whereas in higher surface water contents the adjustment in diffusive resistance around evaporating pores could accommodate the increase in input energy per evaporating pore without significant modifications of surface temperature. These nuanced adjustments are captured by PCEB but not by models that homogenize the evaporating surface and enforce energy balance. 


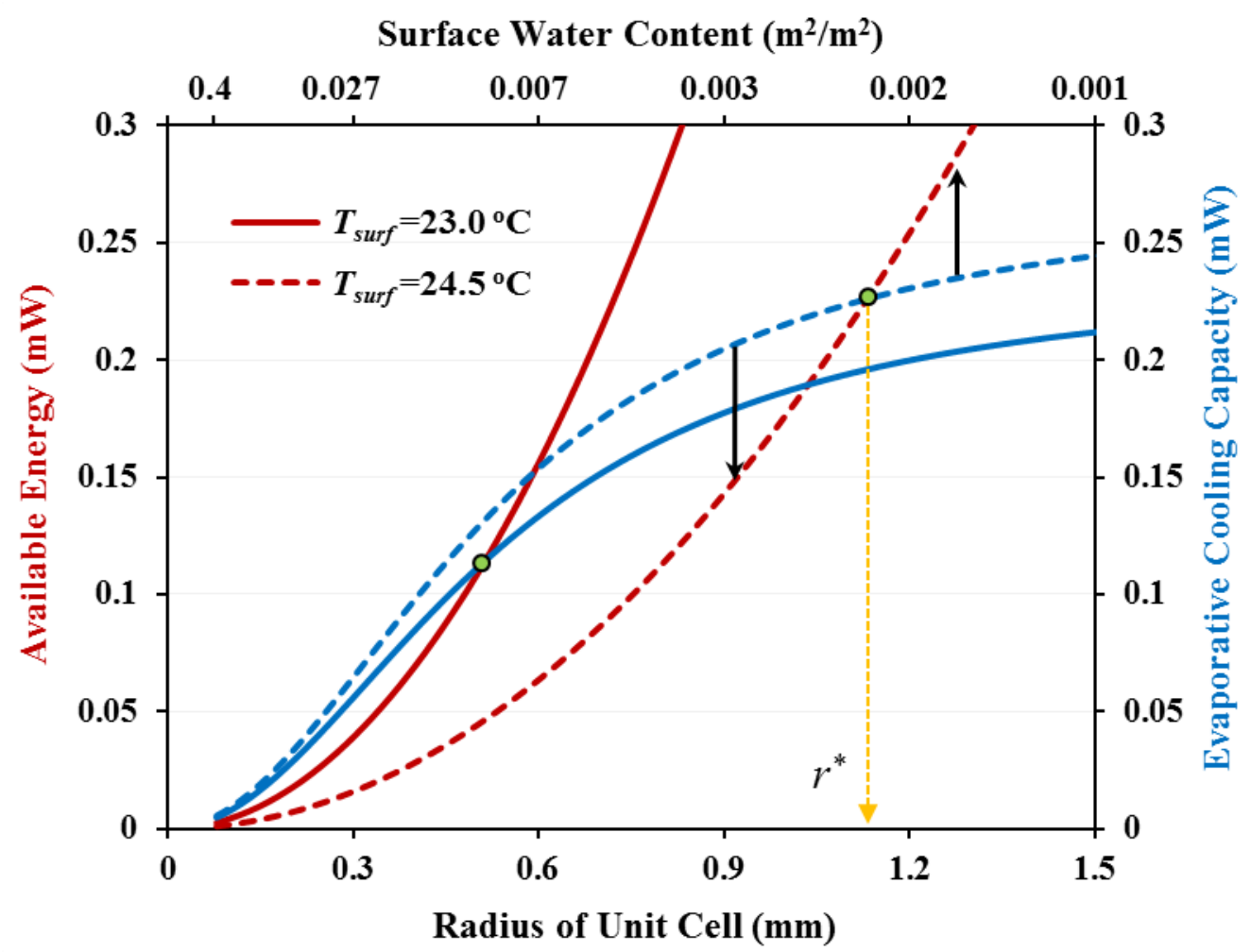

Figure B1. Variation of the available energy for evaporation (left axis) and evaporative cooling capacity (right axis) with radius of unit cell (and corresponding water content) for two different surface temperatures assuming $T_{a}=20^{\circ} \mathrm{C}, R H=50 \%, R_{S}=300 \mathrm{~W} / \mathrm{m}^{2}, u=1 \mathrm{~m} / \mathrm{s}$, and pore radius of $50 \mu \mathrm{m}$. The intersection of the curves marked by symbols depicts unique dry region radius around the representative pore where surface energy balance closure occurs.

\section{Appendix C: Linking pore size distribution with mean surface temperature}

The predictable pore emptying sequence inferred from pore geometry highlights the potential applicability of PCEB model to link pore size distribution with variation of mean surface temperature. We thus considered three systems with different ranges of pore size distributions inferred from the capillary pressure curves (Figure C1) based on the van-Genuchten model [32]:

$$
\Theta=\frac{\theta-\theta_{r}}{\theta_{s}-\theta_{r}}=\left(\frac{1}{1+(\alpha h)^{n}}\right)^{1-1 / n}
$$


where $\Theta$ is effective saturation, $\theta_{s}$ and $\theta_{r}$ are saturated and residual water content, respectively, $\alpha$ and $n$ are van-Genuchten parameters, and $h$ is the capillary head:

$$
h=\frac{2 \sigma}{\rho g a}
$$

in which $\sigma$ and $\rho$ are surface tension and water density, respectively, $g$ is the gravitational acceleration, and $a$ is the pore radius. The volumetric pore size distribution function is defined as [33]:

$$
F=\frac{d \theta}{d a}
$$

where $F \times \Delta a$ represents the volume of pores with radii $a$ and $a+\Delta a$ per unit volume of sample. For the van-Genuchten model, $F$ is expressed as (Figure C2):

$$
F=\frac{d \theta}{d a}=\frac{2 \sigma\left(\theta_{s}-\theta_{r}\right)(n-1) \alpha^{n} h^{n-1}}{\rho g a^{2}\left(1+(\alpha h)^{n}\right)^{(2-1 / n)}}
$$

After deducing pore size distribution from capillary pressure curve for each sample, PCEB model is employed to investigate variation of mean surface temperature during gradual drying of samples with different pore size distributions. Hence, the representative pore radius corresponding to each surface liquid content in PCEB model is inferred from the weighted average of remaining active pores (full pores) on the surface:

$$
\bar{a}=\frac{\sum F_{i} a_{i}}{\sum F_{i}}
$$

Results in Figure C3 demonstrate that main difference emerges for low surface liquid contents where most of surface pores are invaded by the air. Thus, for similar boundary conditions and 
surface liquid content, a surface with pore size distribution dominated by smaller pores would produce a higher evaporative flux that, in turn, will induce lower mean surface temperature relative to surfaces with larger pore sizes as the dominant fraction.

In consideration of the full picture of surface evaporation behavior, one must consider that porous media with different pore size distributions would have different characteristic evaporative lengths [6] which determines the cumulative mass loss during stage 1 (or its duration) and thus affects mean surface temperature especially in the regional context of a patchwork of surfaces with different properties.

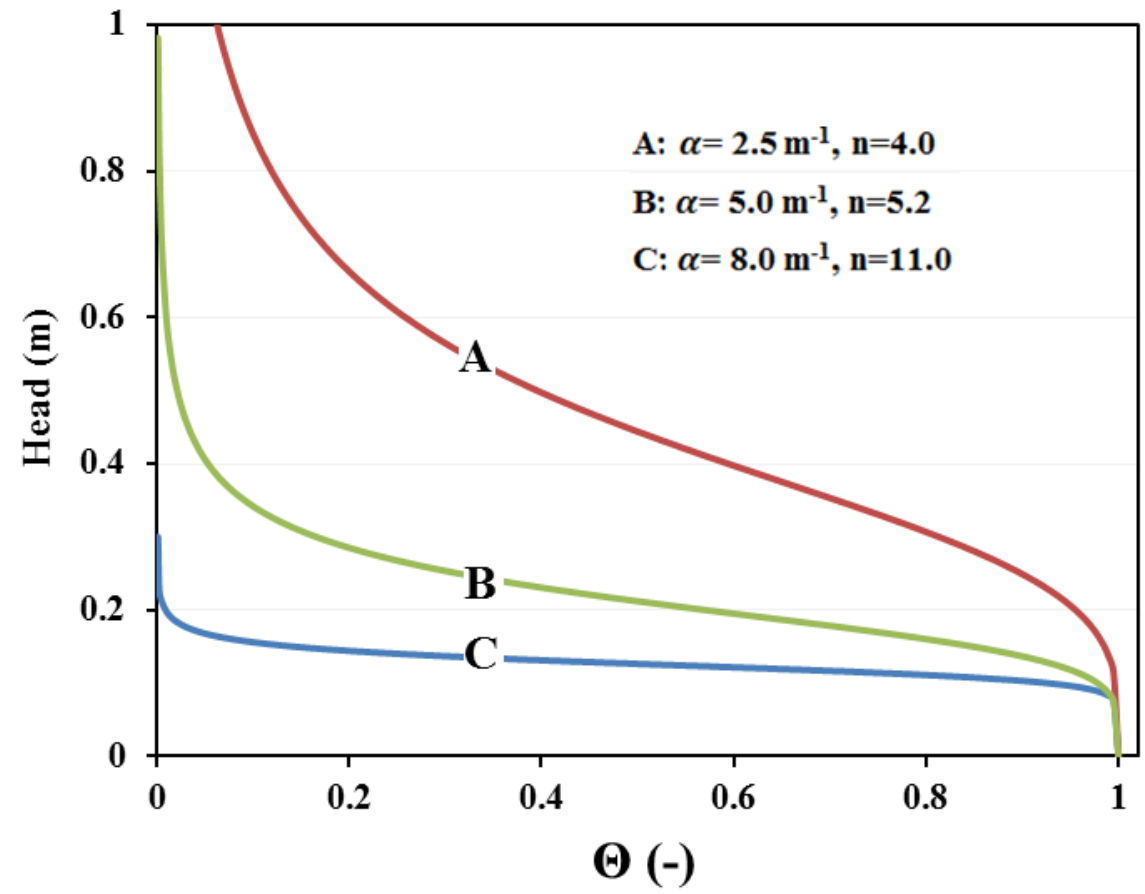

Figure C1. Capillary pressure curve of three different samples deduced from van-Genuchten model assuming $\theta_{s}=0.4 \mathrm{~m}^{3} / \mathrm{m}^{3}$ and $\theta_{r}=0 \mathrm{~m}^{3} / \mathrm{m}^{3}$. 


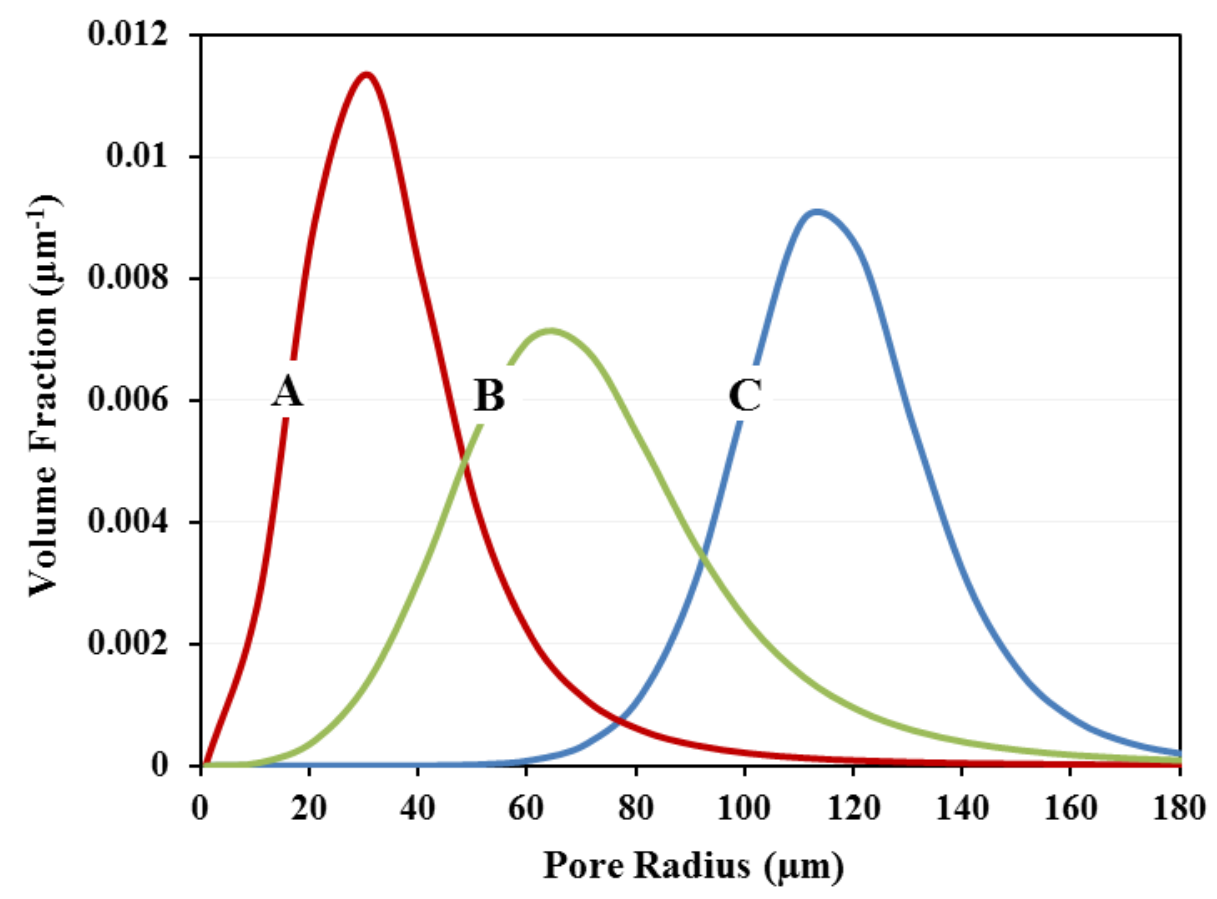

Figure C2. Pore size distribution extracted from capillary pressure curve. 

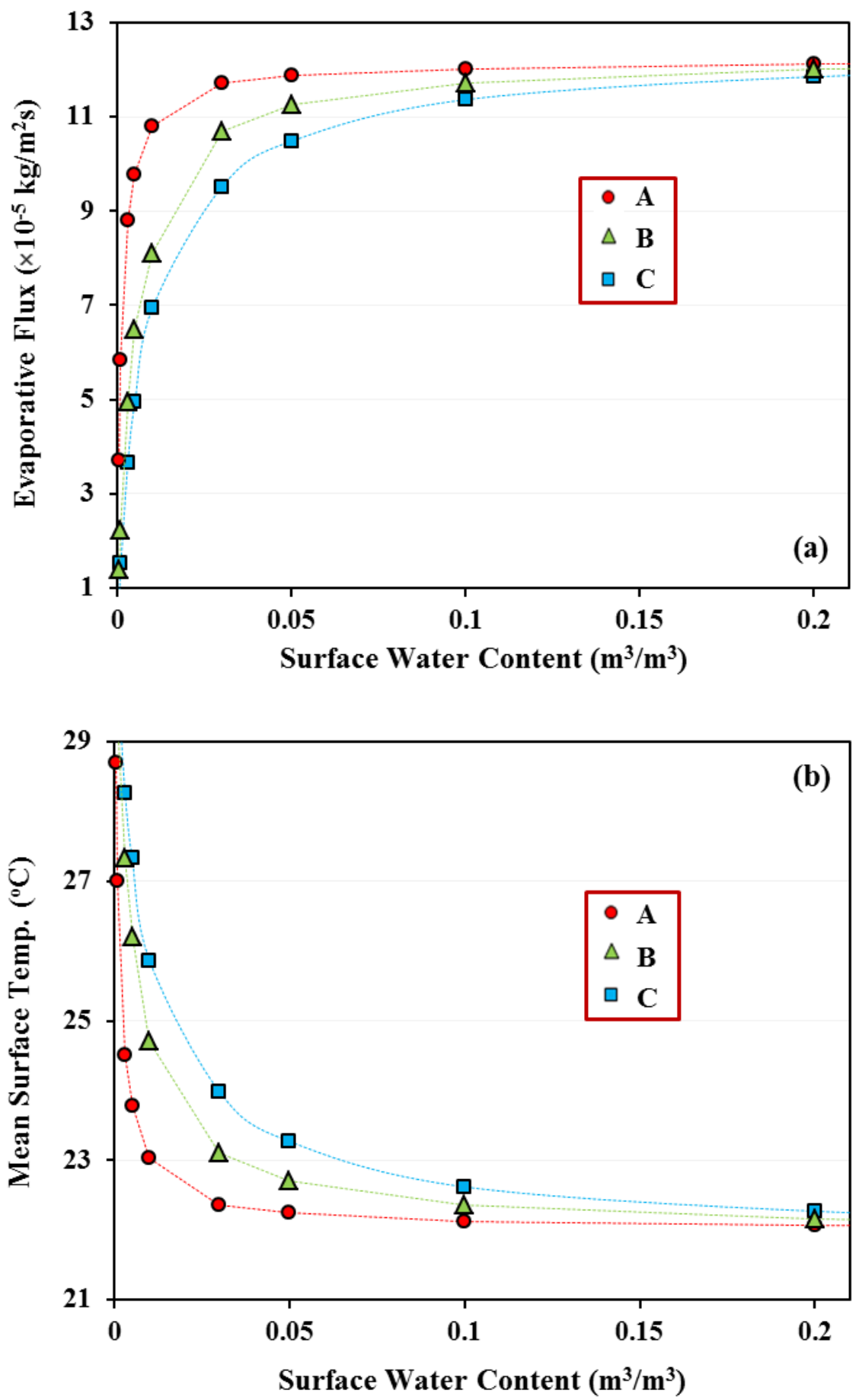

Figure C3. Model predictions of the variation of evaporative flux (a) and mean surface temperature (b) during surface drying. $T_{a}=25^{\circ} \mathrm{C}, R H=50 \%, R_{S}=200 \mathrm{~W} / \mathrm{m}^{2}, u=2 \mathrm{~m} / \mathrm{s}$. 


\section{References}

[1] G.W. Scherer, D.M. Smith, Cavitation during drying of a gel, J. Non. Cryst. Solids. 189 (1995) 197-211. doi:10.1016/0022-3093(95)00222-7.

[2] M. Prat, Recent advances in pore-scale models for drying of porous media, Chem. Eng. J. 86 (2002) 153-164. doi:10.1016/S1385-8947(01)00283-2.

[3] T.M. Shaw, Drying as an immiscible displacement process with fluid counterflow, Phys. Rev. Lett. 59 (1987) 1671-1674. doi:10.1103/PhysRevLett.59.1671.

[4] P. Lehmann, D. Or, Evaporation and capillary coupling across vertical textural contrasts in porous media, Phys. Rev. E - Stat. Nonlinear, Soft Matter Phys. 80 (2009) 1-13. doi:10.1103/PhysRevE.80.046318.

[5] N. Shokri, P. Lehmann, P. Vontobel, D. Or, Drying front and water content dynamics during evaporation from sand delineated by neutron radiography, Water Resour. Res. 44 (2008) 1-11. doi:10.1029/2007WR006385.

[6] P. Lehmann, S. Assouline, D. Or, Characteristic lengths affecting evaporative drying of porous media, Phys. Rev. E - Stat. Nonlinear, Soft Matter Phys. 77 (2008) 1-16. doi:10.1103/PhysRevE.77.056309.

[7] J. van Brakel, Mass transfer in convective drying, 7 ed., Hemisphere, New York, 1980.

[8] A.G. Yiotis, I.N. Tsimpanogiannis, A.K. Stubos, Y.C. Yortsos, Pore-network study of the characteristic periods in the drying of porous materials., J. Colloid Interface Sci. 297 (2006) 738-48. doi:10.1016/j.jcis.2005.11.043.

[9] N. Shokri, D. Or, What determines drying rates at the onset of diffusion controlled stage-2 evaporation from porous media?, Water Resour. Res. 47 (2011) 1-8. doi:10.1029/2010WR010284. 
[10] D. Or, P. Lehmann, E. Shahraeeni, N. Shokri, Advances in Soil Evaporation PhysicsA Review, Vadose Zo. J. 12 (2013) 0. doi:10.2136/vzj2012.0163.

[11] E.U. Schlünder, On the mechanism of the constant drying rate, Chem. Eng. Sci. 43 (1988) 2685-2688.

[12] E. Shahraeeni, P. Lehmann, D. Or, Coupling of evaporative fluxes from drying porous surfaces with air boundary layer: Characteristics of evaporation from discrete pores, Water Resour. Res. 48 (2012) 1-15. doi:10.1029/2012WR011857.

[13] E. Haghighi, E. Shahraeeni, P. Lehmann, D. Or, Evaporation rates across a convective air boundary layer are dominated by diffusion, Water Resour. Res. 49 (2013) 16021610. doi:10.1002/wrcr.20166.

[14] T. Metzger, E. Tsotsas, Influence of Pore Size Distribution on Drying Kinetics: A Simple Capillary Model, Dry. Technol. 23 (2005) 1797-1809. doi:10.1080/07373930500209830.

[15] M. Aminzadeh, D. Or, Temperature dynamics during nonisothermal evaporation from drying porous surfaces, Water Resour. Res. 49 (2013) 7339-7349. doi:10.1002/2013WR014384.

[16] C. Höhmann, P. Stephan, Microscale temperature measurement at an evaporating liquid meniscus, Exp. Therm. Fluid Sci. 26 (2002) 157-162. doi:10.1016/S08941777(02)00122-X.

[17] C. Buffone, K. Sefiane, Investigation of thermocapillary convective patterns and their role in the enhancement of evaporation from pores, Int. J. Multiph. Flow. 30 (2004) 1071-1091. doi:10.1016/j.ijmultiphaseflow.2004.05.010.

[18] C. Buffone, K. Sefiane, W. Easson, Marangoni-driven instabilities of an evaporating 
liquid-vapor interface, Phys. Rev. E - Stat. Nonlinear, Soft Matter Phys. 71 (2005) 310. doi:10.1103/PhysRevE.71.056302.

[19] H. Wang, J.Y. Murthy, S. V. Garimella, Transport from a volatile meniscus inside an open microtube, Int. J. Heat Mass Transf. 51 (2008) 3007-3017. doi:10.1016/j.ijheatmasstransfer.2007.09.011.

[20] F. Chauvet, S. Cazin, P. Duru, M. Prat, Use of infrared thermography for the study of evaporation in a square capillary tube, Int. J. Heat Mass Transf. 53 (2010) 1808-1818. doi:10.1016/j.ijheatmasstransfer.2010.01.008.

[21] K. Sefiane, C.A. Ward, Recent advances on thermocapillary flows and interfacial conditions during the evaporation of liquids., Adv. Colloid Interface Sci. 134-135 (2007) 201-23. doi:10.1016/j.cis.2007.04.020.

[22] M. Aminzadeh, D. Or, Energy partitioning dynamics of drying terrestrial surfaces, J. Hydrol. 519 (2014) 1257-1270.

[23] E. Shahraeeni, D. Or, Quantification of subsurface thermal regimes beneath evaporating porous surfaces, Int. J. Heat Mass Transf. 54 (2011) 4193-4202. doi:10.1016/j.ijheatmasstransfer.2011.05.024.

[24] F.P. Incropera, D.P. DeWitt, Fundamentals of heat and mass transfer, 5 ed., Wiley, New York, 2001.

[25] R. Brodkey, H. Hershey, Transport Phenomena: A Unified Approach, McGraw-Hill, 2003.

[26] F.M. White, Fluid mechanics, McGraw-Hill, New York, 2002.

[27] E. Haghighi, D. Or, Evaporation from porous surfaces into turbulent airflows: Coupling eddy characteristics with pore scale vapor diffusion, Water Resour. Res. 49 
(2013) 8432-8442. doi:10.1002/2012WR013324.

[28] E. Shahraeeni, D. Or, Thermo-evaporative fluxes from heterogeneous porous surfaces resolved by infrared thermography, Water Resour. Res. 46 (2010). doi:10.1029/2009WR008455.

[29] P. Lehmann, D. Or, Effect of wetness patchiness on evaporation dynamics from drying porous surfaces, Water Resour. Res. 49 (2013) 8250-8262. doi:10.1002/2013WR013737.

[30] P.W.J. Glover, E. Walker, Grain-size to effective pore-size transformation derived from electrokinetic theory, Geophysics. 74 (2009) E17. doi:10.1190/1.3033217.

[31] J.A. Dean, Lange's handbook of chemistry, 15 ed., McGraw-Hill, New York, 1999.

[32] M.T. van Genuchten, A Closed-form Equation for Predicting the Hydraulic Conductivity of Unsaturated Soils, Soil Sci. Soc. Am. J. 44 (1980) 892. doi:10.2136/sssaj1980.03615995004400050002x.

[33] K. Kosugi, J.W. Hopmans, J.H. Dane, Methods of Soil Analysis, Part 4, Physical Methods, in: SSSA Book Series, 2002: pp. 728-757. 